\title{
Thermoresponsive Brushes Facilitate Effective Reinforcement of Calcium Phosphate Cements
}

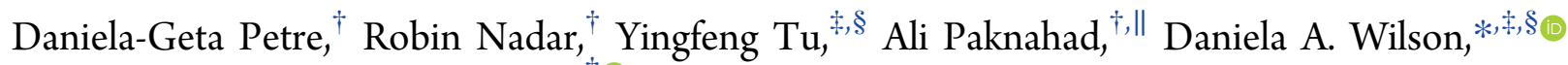 \\ and Sander C. G. Leeuwenburgh* ${ }^{\dagger} \dagger$ \\ ${ }^{\dagger}$ Department of Regenerative Biomaterials, Radboud University Medical Center, 6525 EX Nijmegen, The Netherlands \\ ${ }^{\ddagger}$ Department of Systems Chemistry, Radboud University, 6525 AJ Nijmegen, The Netherlands \\ ${ }^{\S}$ School of Pharmaceutical Science, Guangdong Provincial Key Laboratory of New Drug Screening, Southern Medical University, \\ Guangzhou 510515, China \\ "Department of Computational Mechanics, Faculty of Civil Engineering and Geosciences, Delft University of Technology, $2628 \mathrm{CN}$ \\ Delft, The Netherlands
}

\section{Supporting Information}

ABSTRACT: Calcium phosphate ceramics are frequently applied to stimulate regeneration of bone in view of their excellent biological compatibility with bone tissue. Unfortunately, these bioceramics are also highly brittle. To improve their toughness, fibers can be incorporated as the reinforcing component for the calcium phosphate cements. Herein, we functionalize the surface of poly(vinyl alcohol) fibers with thermoresponsive poly $(\mathrm{N}$-isopropylacrylamide) brushes of tunable thickness to improve simultaneously fiber dispersion

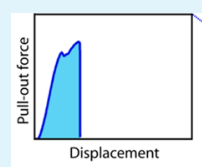
and fiber-matrix affinity. These brushes shift from hydrophilic to hydrophobic behavior at temperatures above their lower critical solution temperature of $32{ }^{\circ} \mathrm{C}$. This dual thermoresponsive shift favors fiber dispersion throughout the hydrophilic calcium phosphate cements $\left(\right.$ at $21{ }^{\circ} \mathrm{C}$ ) and toughens these cements when reaching their hydrophobic state (at $\left.37^{\circ} \mathrm{C}\right)$. The reinforcement efficacy of these surface-modified fibers was almost double at 37 versus $21{ }^{\circ} \mathrm{C}$, which confirms the strong potential of thermoresponsive fibers for reinforcement of calcium phosphate cements.

KEYWORDS: poly(vinyl alcohol) fibers, poly(N-isopropylacrylamide), calcium phosphate cements, reinforcement, interface

\section{INTRODUCTION}

Calcium phosphate cements (CPCs) initially emerged in the 1980s. Since then, these injectable biomaterials have been applied as synthetic bone substitutes in view of their excellent osteocompatibility and clinical handling properties. Their injectability and self-setting properties allow for complete filling of irregularly shaped bone defects, thereby facilitating minimally invasive surgical procedures.

Unfortunately, CPCs are very brittle since these biomaterials are used without any sintering treatment. Consequently, CPCs exhibit low fracture toughness values, ${ }^{1}$ which impede their use in load-bearing skeletal sites. ${ }^{2}$ Therefore, successful development of tough CPCs would expand their clinical applicability toward heavily loaded sites in the spine or even segmental bone defects of long bones. ${ }^{3}$

Several strategies have been explored to improve the mechanical strength of CPCs such as (i) reduction of the porosity of the CPC matrix, ${ }^{4,5}$ (ii) design of a dual-setting system, ${ }^{6}$ or (iii) incorporation of fibers as a reinforcing phase. So far, fiber reinforcement has provided the most efficient results with respect to toughening of CPCs. This idea was inspired by civil engineering, where metallic or polymeric fibers are frequently used to reinforce cementitious matrices.
Multiple key parameters control the process of fiber reinforcement of brittle CPCs such as the type, the aspect ratio, the amount, the mechanical properties, the dispersion, and the fiber-matrix affinity of fibers. Many of these parameters have been previously investigated by incorporating either degradable ${ }^{8,9}$ or nondegradable fibers ${ }^{10,11}$ or by studying the effect of the fiber aspect ratio ${ }^{12}$ or fiber amount relative to the cement matrix ${ }^{13}$ on fiber reinforcement efficacies. Nevertheless, the effect of the fiber-matrix affinity on reinforcement of CPCs has not been investigated in detail in the biomaterials community, although it is widely recognized that this parameter is crucial for reinforcement of engineering materials such as fiber-reinforced concrete. ${ }^{14,15}$ Generally, a too strong bond between the fibers and the matrix will result in fiber rupture with insufficient energy dissipation, whereas a bond that is too weak leads to premature fiber pullout, thereby compromising energy dissipation and, ultimately, the toughening of CPCs. ${ }^{16}$ Only a few studies ${ }^{17,18}$ investigated the effect of fiber-matrix affinity on the mechanical performance of CPCs,

Received: May 17, 2019

Accepted: June 27, 2019

Published: June 27, 2019 

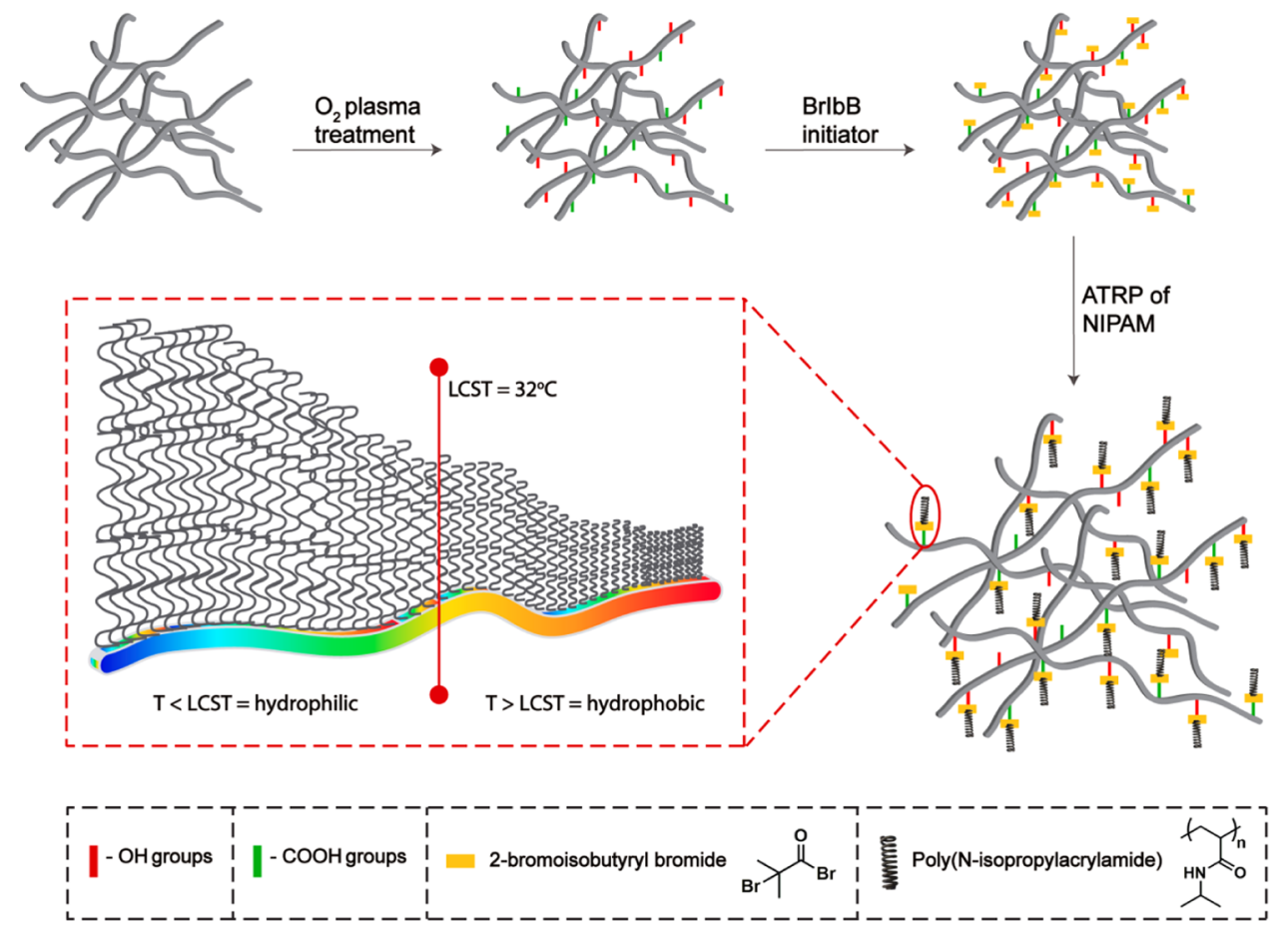

Figure 1. Synthetic pathway to grow PNIPAM brushes onto the surface of PVA fibers.

and these studies did not provide a mechanistic correlation between micromechanical and macroscopic mechanical properties of fiber-reinforced CPC.

So far, degradable polyester fibers have been used for reinforcement of CPCs due to their controllable degradability and the low toxicity of their degradation byproducts. ${ }^{19,20}$ However, they are weak, which compromises their performance as a reinforcing phase in CPC matrices. Additionally, they are hydrophobic, which hinders their dispersion in very hydrophilic CPC matrices. This mismatch in chemical and mechanical properties strongly limits the reinforcement efficacy of these polyester fibers.

In civil engineering, on the other hand, poly(vinyl alcohol) (PVA) fibers are successfully used for the reinforcement of engineered cementitious composites. These fibers are highly suitable for reinforcement of concrete in view of their high tensile strength and modulus, resulting in strongly improved ductility and fracture toughness of PVA fiber-reinforced concrete. ${ }^{21-23}$ However, PVA is a highly hydrophilic polymer due to the abundant presence of hydroxyl groups and, consequently, the affinity of PVA fibers to hydrophilic matrices such as concrete is considered too strong for effective reinforcement. Therefore, PVA fibers are usually covered with hydrophobic oil coatings in civil engineering to reduce the hydrophilicity of PVA fibers and optimize energy dissipation through frictional sliding mechanisms. ${ }^{24}$

Previously, PVA has been added as a soluble nonfibrous additive to calcium phosphate cements, ${ }^{25}$ yet PVA fibers have been used only recently to toughen CPCs in view of their beneficial mechanical properties. ${ }^{26}$ Generally, fiber reinforcement is recognized as a very effective technique to improve the mechanical performance of cementitious matrices, whereas the addition of soluble nonfibrous additives positively affects handling properties ${ }^{27}$ such as injectability or cement cohesion. However, the dispersion and cytocompatibility of the PVA fibers were not yet optimal for biomedical applications.
Therefore, we propose to modify the surface of PVA fibers using covalently attached and biocompatible thermoresponsive poly ( $\mathrm{N}$-isopropylacrylamide) (PNIPAM) brushes. PNIPAM is a thermoresponsive polymer frequently used in biomedicine since it shifts from hydrophilic to hydrophobic behavior above the lower critical solution temperature (LCST) of $32{ }^{\circ} \mathrm{C}$. We hypothesized that the approach of reinforcing CPCs with thermoresponsive PVA fibers is highly relevant since it allows for simultaneous improvement of fiber dispersion and fibermatrix affinity. In their hydrophilic state (below LCST), these fibers would disperse optimally throughout the CPC matrix. On the other hand, in their hydrophobic state (above LCST), these PNIPAM-modified PVA fibers would allow for optimal toughening of the cement matrix through frictional sliding mechanisms.

PNIPAM brushes can be synthesized using atom transfer radical polymerization (ATRP) ${ }^{28}$ which allows for precise control over the molecular weight, grafting density, and thickness of these brushes. ${ }^{29}$ In this way, PNIPAM brushes have been grown onto various substrates including cellulose, ${ }^{30,31}$ clay, ${ }^{32}$ graphene, ${ }^{33,34}$ mesoporous silica, ${ }^{35}$ gold, ${ }^{36}$ titanium, $^{37}$ or even proteins ${ }^{38,39}$ and nanomotors, ${ }^{40}$ thereby allowing for temperature-induced control over the polarity of the coated substrates. Recently, PNIPAM brushes have also been deposited onto nano- and microfibers including lignin and poly(ethylene terephthalate) fibers $^{41-43}$ for possible applications such as separation and purification devices. ${ }^{41}$ So far, PNIPAM brushes have not yet been used to surface-modify fibers for reinforcement of (bio)ceramic matrices.

In the current study, we investigated the effect of PNIPAMmodified microfibers on the mechanical behavior of CPCs. To this end, we have covalently grown PNIPAM brushes with different grafting densities and brush thicknesses onto the surface of PVA fibers by varying the molar ratios among the monomer, ligand, and catalyst. The surface morphology, phase transition temperature, wettability, and molecular weight of the 
modified fibers were analyzed using electron microscopy, differential scanning calorimetry (DSC), contact angle measurements, and gas permeation chromatography, respectively. Next, the modified fibers were incorporated into CPCs followed by mechanical testing the fiber-reinforced composites to quantify the work of fracture (WOF) as a measure of macroscopic toughness and the work of pullout (WOP) as a measure of the micromechanical fiber-matrix affinity.

\section{EXPERIMENTAL SECTION}

2.1. Materials. Commercially available poly(vinyl alcohol) (PVA, Kuralon RFS400) microfibers were kindly supplied by Kuraray Europe GmbH (Hattersheim, Germany) (dimensions and mechanical properties are specified in Table S1). $\alpha$-Tricalcium phosphate $(\alpha$ TCP) powder was provided by CAM Bioceramics (Leiden, The Netherlands). N-Isopropylacrylamide (NIPAM, 97\%, Sigma-Aldrich) was purified by recrystallization twice from toluene/hexane solution $(50 \% \mathrm{v} / \mathrm{v})$ and dried under vacuum for $48 \mathrm{~h}$ at room temperature. Copper(I) bromide (CuBr, 98\% Sigma-Aldrich) was stirred overnight in excessive acetic acid, filtered until the suspension turned pale green, and dried under vacuum at room temperature for $12 \mathrm{~h}^{44} \alpha$ Bromoisobutyryl bromide (BrIbB, 98\%), $N, N, N^{\prime}, N^{\prime \prime}, N^{\prime \prime}$-pentamethyldiethylenetriamine (PMDETA, 99\%), triethylamine (TEA, 99\%), Toluidine Blue $\mathrm{O}(\mathrm{TBO})$, and sodium dihydrogen phosphate dihydrate $\left(\mathrm{NaH}_{2} \mathrm{PO}_{4} \cdot 2 \mathrm{H}_{2} \mathrm{O}\right)$ were purchased from Sigma-Aldrich and used as-received without purification. Methanol ( $\mathrm{MeOH}, 99.9 \%)$ and tetrahydrofuran (THF, 99.9\%) were purchased from Merck.

2.2. PNIPAM Modification of PVA Fibers. 2.2.1. Plasma Treatment and Attachment of the Initiator. The modification scheme for the functionalization of PVA fibers with thermosensitive PNIPAM brushes is schematically depicted in Figure 1. First, fibers were cleaned with a mixture of ethanol and Milli-Q water $\left(\mathrm{H}_{2} \mathrm{O}\right)$ and dried overnight in air. Dried fibers were subjected to an oxygen plasma treatment to create oxygen-containing functional groups for covalent attachment of the $\alpha$-bromoisobutyryl bromide (BrIbB) initiator. Oxygen plasma treatment was then performed by a Femto low-pressure plasma system (Diener electronic GmbH, Germany) operated at $40 \mathrm{kHz}$. The plasma treatment duration was varied between $21 \mathrm{~s}$ and $10 \mathrm{~min}$, whereas the power densities were fixed at either 60 or $90 \mathrm{~W}$. To ensure that the entire fiber surface was activated, both sides of the fibers were pretreated in separate runs. For the attachment of the initiator, plasma-treated PVA fibers were placed in a round-bottom flask, and freshly distilled THF $(40 \mathrm{~mL})$ was added. TEA ( $300 \mu \mathrm{L}, 1: 2$ molar ratio between bromide and TEA) was also added to neutralize the liberated $\mathrm{HBr}$. Subsequently, BrIbB was added dropwise at $0{ }^{\circ} \mathrm{C}$ to the stirring solution at a $1: 4$ molar ratio between carboxyl groups (resulting from oxygen plasma treatment) and the bromide initiator. The flask was flushed with nitrogen before sealing, and the temperature was allowed to reach room temperature. The reaction was carried out overnight, after which the fibers were washed with $\mathrm{MeOH}$ and $\mathrm{H}_{2} \mathrm{O}$. Finally, the fibers were freeze-dried.

2.2.2. ATRP Synthesis of PNIPAM Brushes. NIPAM monomer and PMDETA ligand ( $42 \mu \mathrm{L}, 0.2 \mathrm{mmol}$ ) were added at molar NIPAM/ CuBr-PMDETA ratios of 25:1, 50:1, and 100:1 in a round-bottom flask to a 9:1 mixture of $\mathrm{MeOH} / \mathrm{H}_{2} \mathrm{O}(40 \mathrm{~mL})$. The resulting fibers are referred to as PVA-PNIPAM 25, PVA-PNIPAM 50, and PVAPNIPAM 100, respectively. Subsequently, this monomer/ligand mixture was flushed with argon for $30 \mathrm{~min}$. $\mathrm{CuBr}$ powder $(28.6 \mathrm{mg}$, $0.2 \mathrm{mmol}$ ) was flushed with argon for $30 \mathrm{~min}$ in another flask. A 60 $\mathrm{mL}$ syringe was used to transfer the NIPAM/PMDETA solution to the flask containing the $\mathrm{CuBr}$ catalyst. This solution was stirred for another 30 min and bubbled with argon. ${ }^{44}$ The Br-treated PVA fibers were sealed in flasks and flushed with argon for at least $30 \mathrm{~min}$. Subsequently, the solution containing the NIPAM monomer, the PMDETA ligand, and the $\mathrm{CuBr}$ catalyst was transferred to the flask containing the $\mathrm{Br}$-treated fibers. The polymerization was allowed to proceed for $21 \mathrm{~h}$ at room temperature under an argon atmosphere. To terminate the polymerization reaction, the flask was aerated and fibers were exhaustively rinsed and washed overnight with $\mathrm{MeOH}$ and $\mathrm{H}_{2} \mathrm{O}$ to remove any unreacted polymers followed by freeze-drying. Additionally, both as-received PVA fibers (covered by a hydrophobic coating) and uncoated PVA fibers (stripped off their coating by extraction in THF for $72 \mathrm{~h}$ ) were used as control groups. THF was used to remove the hydrophobic coating from as-received PVA fibers since this solvent was also used to attach the initiator for PNIPAM polymerization onto the PVA fibers.

2.2.3. Cleavage of the PNIPAM Brushes. The grafted PNIPAM brushes were removed from the PVA fibers by reacting each grafted sample ( $25 \mathrm{mg}$ of fibers) with $10 \mathrm{~mL}$ of $2 \mathrm{M} \mathrm{NaOH}$ aqueous solution for 1 week. The supernatant in the solution was removed, and the remaining insoluble PNIPAM was dissolved in $0.2 \mathrm{M} \mathrm{NaOH}$ aqueous solution over the weekend, neutralized with $0.1 \mathrm{M} \mathrm{HCl}$, and dialyzed for 2 days in a dialysis tube with a cutoff of $1000 \mathrm{Da}$. The cleaved PNIPAM was retrieved using freeze-drying.

2.3. Physicochemical Characterization of PNIPAM-Functionalized PVA Fibers. The density of carboxyl groups generated on the PVA fibers after oxygen plasma treatment was determined by the Toluidine Blue O (TBO) assay. ${ }^{45}$ Briefly, plasma-treated PVA fibers were immersed into a $500 \mu \mathrm{M}$ TBO aqueous solution at $\mathrm{pH} 11$, after which the formation of ionic complexes between the carboxyl groups and TBO was allowed to proceed for $2 \mathrm{~h}$ at $37^{\circ} \mathrm{C}$. After rinsing with $\mathrm{NaOH}$ solution and $\mathrm{H}_{2} \mathrm{O}$ twice, the fibers were immersed in $50 \%$ acetic acid solution for $72 \mathrm{~h}$ to remove the bonded TBO. The absorbance at $633 \mathrm{~nm}$ was recorded with a Biotek Synergy HT plate reader. The amount of carboxyl groups was determined using a calibration curve of TBO in 50\% acetic acid solution.

Inductively coupled plasma-optical emission spectroscopy (ICPOES; iCAP 6000, Thermo Fisher Scientific) analysis was performed to determine the bromine and copper contents of the fibers before and after their modification with PNIPAM brushes. This analysis was performed in triplicate for all groups after treating the pristine and surface-modified fibers in a $65 \%$ nitric acid solution for $12 \mathrm{~h}$ followed by dilution of the samples to a $5 \%$ nitric acid solution.

A scanning electron microscope (SEM, JEOL 6310, Jeol Corp.) equipped with an energy-dispersive X-ray (EDX) spectroscopy detector was used to observe the surface morphology of the fibers after polymerization of the PNIPAM brushes. The thickness of the dry PNIPAM brushes was assessed by means of SEM of at least three fiber cross sections per experimental group using ImageJ software (Wayne Rasband, Research Services Branch, National Institute of Mental Health, Bethesda). The interface between the PNIPAM brushes and underlying PVA fibers was highlighted with a purple line to allow for a more precise measurement of brush thickness. Dry thickness measurements were performed at $\sim 100$ points along the fiber surface to provide more information on the reproducibility.

To determine the wettability of the surface-modified fibers, contact angle measurements were executed by the meniscus contact angle technique by means of an optical tensiometer (Attension Theta, Biolin Scientific). The fiber was fixed to a holder and immersed $\sim 2$ mm in either $\mathrm{H}_{2} \mathrm{O}$ or a 4 wt $\% \mathrm{NaH}_{2} \mathrm{PO}_{4}$ solution. The contact angle was measured from the shape of the liquid contacting the fiber. A Young-Laplace fitting was used to calculate the meniscus contact angles. The mean contact angle value for each experimental group represents the average contact angle values of three different fibers $(n$ $=3$ ).

Differential scanning calorimetry (DSC, Mettler Toledo) was used to characterize the thermoresponsive behavior of the PNIPAMmodified PVA fibers using $2 \mathrm{mg}$ of fibers in $20 \mu \mathrm{L}$ of either $\mathrm{H}_{2} \mathrm{O}, 4 \mathrm{wt}$ $\% \mathrm{NaH}_{2} \mathrm{PO}_{4}$, or phosphate-buffered saline (PBS) solution. The onset point of the endothermal peak in the calorimetric curves was used to determine the lower critical solution temperature (LCST). The onset temperature was obtained by the intersection of the baseline and the leading edge of the endotherm. The samples were scanned at $5{ }^{\circ} \mathrm{C} /$ min between 10 and $40{ }^{\circ} \mathrm{C}$, referenced against a medium-filled pan $\left(\mathrm{H}_{2} \mathrm{O}, \mathrm{H}_{2} \mathrm{PO}_{4}\right.$, or $\left.\mathrm{PBS}\right)$. All temperatures were determined from the second heating scan.

To examine the dimensional changes of the thermoresponsive PNIPAM brushes (compaction and expansion) as a function of temperature, the modified fibers were placed in a quartz crucible and 
visualized with a bright-field hotstage microscope (Zeiss Axioplan 2) modified with a hotstage (Linkam LTS420) equipped with a temperature control unit (Linkam T95) and a liquid nitrogen pump for cooling (Linkam LNP95). Images were captured with a video camera (Evolution VF, Media Cybernetics). The fibers were subjected to a heating and cooling cycle between 20 and $40{ }^{\circ} \mathrm{C}$. The thickness of the PNIPAM brush during compaction and expansion in different media was assessed as a function of temperature (below and above the LCST) using the corresponding bright-field micrographs (ImageJ software). Gel permeation chromatography (GPC) was performed to measure the molecular weights of both the hydrophobic coating (extracted from the as-received PVA fibers using THF) and the cleaved PNIPAM brushes. To this end, a Shodex GPC column equipped with UV and differential refractive index detectors $(254,215$ $\mathrm{nm}$ ) was used. THF was used as the eluent, and the flow rate was fixed at $1 \mathrm{~mL} / \mathrm{min}$. Polystyrene standards were used for calibration.

The grafting density (in chains $/ \mathrm{nm}^{2}$ ) was obtained from the following equation

Grafting density:

$$
\sigma=\frac{h \rho N_{\mathrm{A}}}{M_{\mathrm{n}}}
$$

where $h$ corresponds to the dry film thickness of the polymer brush, $\rho$ is the polymer density, $N_{\mathrm{A}}$ is Avogadro's constant, and $M_{\mathrm{n}}$ is the number-average molecular weight of the surface-tethered polymers.

2.4. In Vitro Cytocompatibility of PNIPAM-Modified PVA Fibers. The cytocompatibility of as-received and PNIPAM-modified PVA fibers was investigated by measuring the proliferation of osteoblastic cells (MC3T3-E1, ATCC) in the presence of $10 \mathrm{mg} /$ $\mathrm{mL}$ fibers (diameter $200 \mu \mathrm{m}$, length $8.8 \mathrm{~mm}$ ). Fibers were weighed in Eppendorf tubes and washed twice with $70 \%$ ethanol (v/v) for $15 \mathrm{~min}$ per washing step. The fibers were transferred into Transwell permeable supports ( $4 \mu \mathrm{m}$ pore size, Merck $\mathrm{KGaA})$ followed by two washing cycles using sterile PBS. MC3T3-E1 cells were cultured in $\alpha$-minimum essential medium containing $10 \%$ fetal bovine serum and $1 \%$ penicillin (Gibco, Life Technologies). Cells were seeded at a density of 10000 cells $/ \mathrm{cm}^{2}$ in 12 -well plates. Subsequently, the transwell supports containing the fibers were placed in the wells ensuring complete submersion of the fibers in the medium. Cells were cultured for 1,3 , and 7 days (medium was replaced after 3 days) in an incubator with $5 \% \mathrm{CO}_{2}$ at $37^{\circ} \mathrm{C}$. After each time point, the medium and transwell supports were discarded and cells were washed twice with $\mathrm{PBS}$, followed by addition of $\mathrm{H}_{2} \mathrm{O}(1 \mathrm{~mL}$ per well $)$ and two freeze-thaw cycles. The cellular DNA content was measured using a Quant-IT PicoGreen ds-DNA assay kit (Invitrogen). In brief, samples or standard solution $(100 \mu \mathrm{L})$ were added to a working solution in a 96-well plate. The plates were then incubated for $5 \mathrm{~min}$ in the dark at room temperature, and the fluorescence emission was read (excitation: 485/20 nm; emission: 530/25 nm). The DNA content of the experimental groups was expressed relative to the DNA content of cell growth in the absence of PVA fibers (positive control).

2.5. Preparation of Fiber-Reinforced Calcium Phosphate Cements. The solid powder phase ( $\alpha$-TCP supplemented with PVA fibers) was manually mixed with the liquid phase $(4 \% \mathrm{w} / \mathrm{v}$ $\mathrm{Na}_{2} \mathrm{HPO}_{4}$ ), resulting in fiber-reinforced calcium phosphate cement samples at a liquid/powder $(\mathrm{L} / \mathrm{P})$ ratio of 1:2. The weight fraction of the PVA fibers in the cement was fixed at $2.5 \mathrm{wt} \%$. The length of the fibers was fixed at an average of $8.8 \mathrm{~mm}$ (aspect ratio of 44) by manual cutting prior to PNIPAM modification of the fibers. The frequency distribution of fiber lengths (Figure S1) after manual cutting was assessed by measuring $\geq 100$ fibers using Image J software. After manual mixing of the solid and liquid phases, the resulting pastes were placed in rectangular $10 \times 10 \times 40 \mathrm{~mm}^{3}$ poly(dimethylsiloxane) molds and allowed to set at room temperature for $12 \mathrm{~h}$. Subsequently, the hardened fiber-reinforced composites were immersed in PBS for 3 days at $37{ }^{\circ} \mathrm{C}$ to allow for conversion of $\alpha$ TCP to hydroxyapatite.

2.6. Mechanical Properties of the Fiber-Reinforced CPCs. 2.6.1. Bulk Mechanical Properties. Fiber-free and fiber-reinforced
CPC specimens were subjected to three-point flexural testing at different temperatures (in PBS of either 21 or $37^{\circ} \mathrm{C}$ ) using a tensile bench (LS5, Lloyd Instruments, Ametek), a flexure span of $30 \mathrm{~mm}$, and a $1 \mathrm{kN}$ load cell. These three-point flexural tests were used to determine the flexural strength, flexural modulus, and work of fracture (WOF, a measure of toughness) from the load-displacement curves Flexural strength:

$$
S_{\mathrm{c}}=\frac{3 P_{\max } L}{2 b h^{2}}
$$

where $P_{\max }$ is the maximum load on the load-displacement curve, $L$ is the support span, $b$ is the specimen width, and $h$ is the specimen thickness.

Flexural modulus:

$$
E_{\text {bend }}=\frac{m L^{3}}{4 b h^{3}}
$$

where $m$ is the slope of tangent to the initial straight-line portion of the load-displacement curve.

Work of fracture:

$$
\mathrm{WOF}=\frac{A}{b h}
$$

where $A$ represents the area under the load-displacement curve.

For a consistent calculation of the WOF of the fiber-reinforced composite specimens, the test was stopped at a crosshead displacement of $4 \mathrm{~mm}$, and the area under the load-displacement curves was integrated to quantify the WOF values.

2.6.2. Single-Fiber Pullout Test. To perform single-fiber pullout tests, PVA fibers with a length of $18 \mathrm{~mm}$ (aspect ratio 90) were embedded at an embedment length of $8 \mathrm{~mm}$ along the central longitudinal axis of a cylindrical CPC specimen $(6 \mathrm{~mm}$ diameter, 8 $\mathrm{mm}$ height). To this end, molds were three-dimensional (3D)-printed from water-soluble PVA using an Ultimaker 2+ 3D-printer (Ultimaker Co., Ltd.) to allow for full hardening of the specimens. These watersoluble molds were designed with a groove in the middle to align the embedded fiber along the longitudinal central axis of the CPC cylinder. The solid powder and liquid phase as described above $(\mathrm{L} / \mathrm{P}$ ratio of 0.5 ) were mixed, and the resulting paste was transferred into the dissolvable molds. Before cement hardening, a single PVA fiber was placed in the grooves of the mold, and the sample was allowed to harden overnight. Subsequently, the samples were placed in $\mathrm{H}_{2} \mathrm{O}$ at $37^{\circ} \mathrm{C}$ to dissolve the mold and allow for hardening, after which the composites were incubated in PBS for 3 days at $37{ }^{\circ} \mathrm{C}$ to allow for complete hardening.

The single-fiber pullout tests were performed using an in-housedeveloped testing method. Briefly, the CPC cylinder containing the single fiber was glued (Pleximon glue) to a $3 \mathrm{D}$-printed rectangular block made of polylactic acid (Silver Metallic $2.85 \mathrm{~mm}$ filament), whereas the protruding end of the embedded fiber was glued (Pattex Plastics) to an aluminum metal sheet. The block and aluminum sheet were mounted to the clamping fixtures of the tensile bench. Subsequently, the fiber was pulled out at a crosshead speed of 1 $\mathrm{mm} / \mathrm{min}$ using a tensile bench (LS5, Lloyd Instruments, Ametek) equipped with a $100 \mathrm{~N}$ load cell. The test was completed at a displacement of $8 \mathrm{~mm}$, and the work of pullout (WOP) values were calculated by integrating the area under the load-displacement curves. The average interfacial shear strength (IFSS) of the fibers and the cement matrix was approximated as follows

Interfacial shear strength:

$$
\tau=\frac{P_{\max }}{\pi d_{\mathrm{f}} l_{\mathrm{f}}}
$$

where $P_{\max }$ is the maximum pullout load, $d_{\mathrm{f}}$ is the fiber diameter, and $l_{\mathrm{f}}$ is the fiber embedment length $(8 \mathrm{~mm})$. To investigate the pullout behavior as a function of temperature, the specimens were tested 
under liquid conditions in PBS at either 21 or $37^{\circ} \mathrm{C}(n=15)$. An inhouse testing method was developed to allow for testing of the modified fibers under liquid conditions. Testing was conducted in a chamber filled with PBS at either room temperature $\left(21^{\circ} \mathrm{C}\right)$ or at 37 ${ }^{\circ} \mathrm{C}$. The temperature variation for each tested sample was in the range of $\sim 2{ }^{\circ} \mathrm{C}$, as measured by a digital thermometer.

2.7. X-ray Diffraction (XRD). After flexural testing, solid samples were ground to powder, and the phase compositions of fiber-free and fiber-reinforced $\mathrm{CPC}$ specimens were analyzed using an X-ray diffraction (XRD) system (X'Pert3 PANalytical, Philips). The measurements were performed at $45 \mathrm{kV}$ and $30 \mathrm{~mA}$, and the scan was obtained from 20 to $50^{\circ} 2 \theta$.

2.8. Morphological Assessment and Nanocomputed Tomography (Nano-CT). The surface of the fibers and the cross section of the fractured composite samples were morphologically assessed using SEM. To this end, samples were sputter-coated twice with a $10 \mathrm{~nm}$ chromium layer and imaged at $5 \mathrm{kV}$. To image fiber dispersion and specimen porosity of the CPCs, the composite samples were scanned by using a nanocomputed tomography (nano-CT) imaging system (NanoTom Phoenix, General Electric). Samples were recorded using a voxel size of $5.9 \mu \mathrm{m}$, with an X-ray source of $90 \mathrm{kV}$ and a current of $110 \mu \mathrm{A}$. The exposure time was $500 \mathrm{~ms}$ without the application of a filter. The obtained projected files were analyzed with CT-Analyzer software (version 1.17.7.2+, Bruker), and the 3D visualization was performed with CTvox software (version 3.0.0 r1114, Bruker).

2.9. Statistical Analysis. All data were expressed as mean \pm standard deviation. For the chemical assays, one-way analysis of variance (ANOVA) followed by a Bonferroni post hoc test was applied. The pullout values were analyzed using Student's $t$-test, whereas the mechanical properties were analyzed statistically by means of two-way ANOVA followed by a Tukey post hoc test. For all tests, the level of significance was set at $p<0.05$. All statistical tests were executed using GraphPad Prism software (GraphPad Software, Inc.).

\section{RESULTS AND DISCUSSION}

The efficacy of fiber reinforcement of ceramic matrices is partially determined by the fiber-matrix interface, but this crucial parameter has hardly been addressed so far in the research dedicated to fiber-reinforced bioceramic materials. Herein, poly(vinyl alcohol) fibers were selected for reinforcement of CPCs in view of their high strength and stiffness, whereas poly $(\mathrm{N}$-isopropylacrylamide $)$ brushes were surfacetethered onto the PVA fibers in view of their thermoresponsive behavior. We hypothesized that reinforcement of CPCs with thermoresponsive PVA fibers allows for simultaneous improvement of fiber dispersion and fiber-matrix affinity. Below their LCST, these fibers would disperse optimally throughout the CPC matrix, whereas in their hydrophobic state, above their LCST, these PNIPAM-modified PVA fibers would allow for toughening of the cement matrix through frictional sliding mechanisms.

3.1. Physicochemical Characterization of PNIPAMFunctionalized PVA Fibers. Low-pressure oxygen plasma treatment is often used to improve the wettability of surfaces and introduce functional groups for further chemical functionalization. Usually, hydroperoxides are formed during oxygen plasma treatment, followed by their decomposition and formation of highly reactive hydroxyl and alkyl radicals. Finally, polar groups such as hydroxyl, carbonyl, and carboxyl groups are formed on the surface of the substrate due to the removal of hydrogen atoms from the polymer chain. ${ }^{46}$ To investigate the effect of power density and plasma treatment on the formation of carboxyl groups, we quantified the density of carboxyl groups at the surface of PVA fibers using the TBO assay. ${ }^{45}$ Figure $2 \mathrm{a}$ shows that the amount of carboxyl groups resulting from plasma treatment did not depend on the power

\section{a}
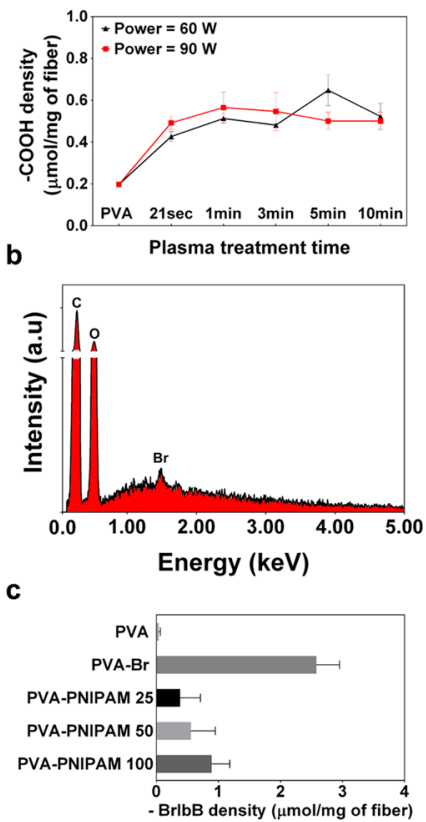

Figure 2. (a) Density of carboxyl groups measured on PVA fibers, (b) EDX spectrum of the Br-treated PVA fiber, (c) bromine content measured after attachment of the BrIbB initiator to PVA fibers and the remaining bromine content after the ATRP reaction.

density or treatment duration, leading to a saturation of the surface with oxygen-containing functional groups.

Based on these results, we used a power of $60 \mathrm{~W}$ and a plasma duration time of $21 \mathrm{~s}$ to prevent damage to the fibers while ensuring the formation of active functionalities at the fiber surface from which the brominated initiator will be conjugated. Figure $2 \mathrm{~b}$ presents an EDX spectrum of the fiber surface after attachment of the BrIbB initiator, which confirms the presence of bromine. The amount of BrIbB after this reaction was quantified using ICP-OES analysis, and it reached values of up to $2.5 \mu \mathrm{mol} \mathrm{BrIbB} / \mathrm{mg}$ of fiber (Figure 2c, PVA$\mathrm{Br})$. The measured molar excess of bromine relative to carboxyl groups confirmed that covalent attachment of the BrIbB initiator was highly efficient. This excess of bromine can be attributed to the presence of oxygen-containing functional groups, which were not detected by the TBO assay such as carbonyl and hydroxyl groups. As observed previously by other groups, the concentration of carbonyl and carboxyl groups remains constant, whereas the concentration of hydroxyl groups increases with increasing plasma time. ${ }^{47}$ Figure $2 \mathrm{c}$ also presents the bromine content after the polymerization reaction for the modified PNIPAM-PVA fibers. Evidently, the highest bromine content after polymerization was present on the PVAPNIPAM 100 fibers $(\sim 0.9 \mu \mathrm{mol} / \mathrm{mg}$ of fiber $)$.

As shown in Figure 3a,b, the surface of pristine PVA fibers was smooth with only few irregularities present at the fiber surface. The surface morphology of the PNIPAM-modified fibers, on the other hand, was considerably roughened due to the formation of polymeric brushes from the fiber surface following the ATRP reaction (Figure $3 c-h$ ).

The amount of polymeric brushes grown at a molar NIPAM/CuBr-PMDETA ratio of 25:1 (Figure 3c,d) was 


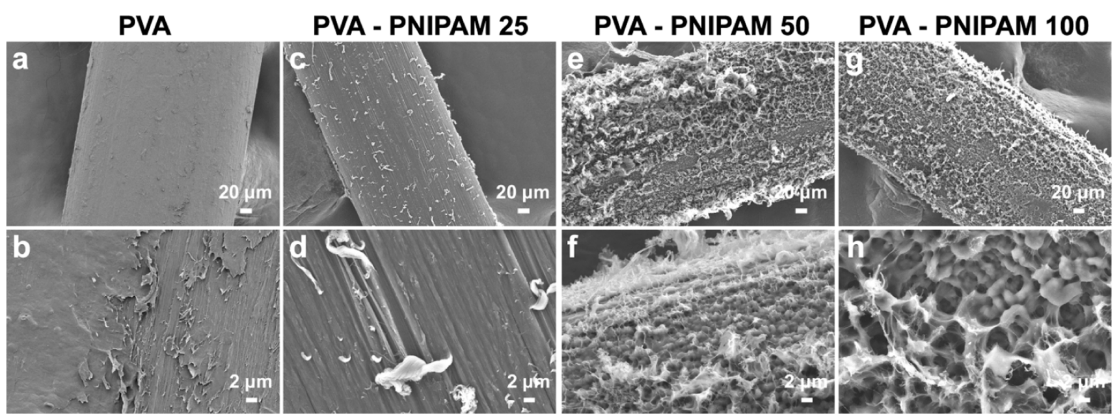

Figure 3. Scanning electron micrographs of pristine and modified fibers at two different magnifications: (a, b) pristine PVA fibers, (c, d) PVAPNIPAM 25-modified fibers, (e, f) PVA-PNIPAM 50-modified fibers, and (g, h) PVA-PNIPAM 100-modified fibers.

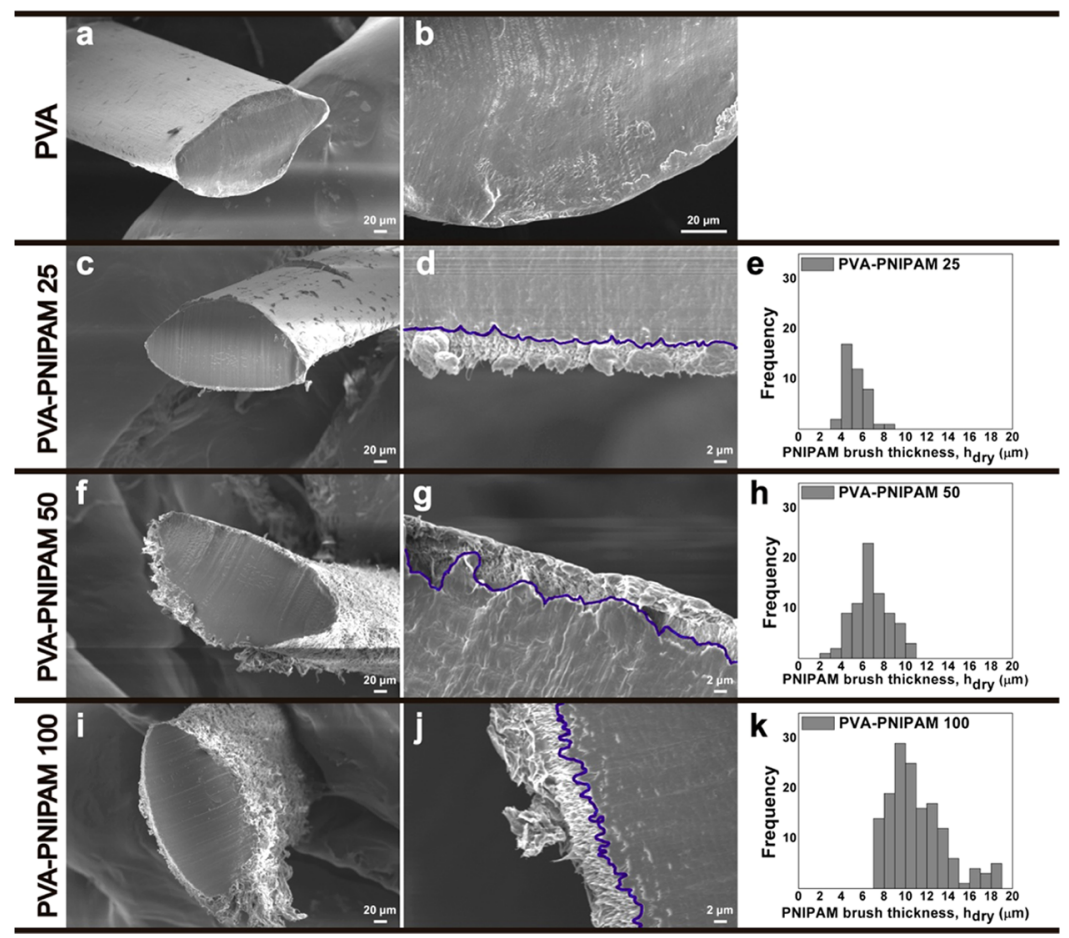

Figure 4. Scanning electron micrographs of cross sections of pristine and PNIPAM-modified fibers at low and high magnifications as well as the histogram of the thickness of dry PNIPAM brushes. ( $a, b)$ pristine PVA fibers, (c-e) PVA-PNIPAM 25 fibers, (f-h) PVA-PNIPAM 50 fibers, and (i-k) PVA-PNIPAM 100 fibers; the separation between PNIPAM brushes and the fiber is marked by the purple line.

lower compared with fibers prepared at higher NIPAM/CuBrPMDETA ratios of 50:1 and 100:1. The brushes were homogeneously distributed over the fiber surface. At higher molar ratios of 50 and 100 (Figure $3 \mathrm{e}-\mathrm{h}$ ), the fibers were fully covered with polymeric brushes, resulting in a much rougher fiber surface and increased diameter compared with their pristine counterparts.

To investigate the difference between the thicknesses of the polymeric layers grown on the fiber surface at different molar ratios, we analyzed the fiber cross sections of the pristine and modified fibers. The cross section of pristine PVA fibers (Figure 4a,b) showed a sharp fiber edge, whereas the surface modification using PNIPAM brushes was clearly visible using SEM. The interface between the PNIPAM brushes and underlying PVA fibers was highlighted with a purple line to allow for a more precise measurement of brush thickness.

For the PVA-PNIPAM 25 sample, a brush thickness of approximately $5 \pm 0.95 \mu \mathrm{m}$ was observed (Figure $4 \mathrm{c}, \mathrm{d}$ ), which increased with increasing NIPAM/CuBr-PMDETA molar ratio to about $11 \pm 2.7 \mu \mathrm{m}$ for the PVA-PNIPAM 100 fibers (Figure
$4 i, j)$. The dry thickness of the PNIPAM brush was heterogeneous across the fiber surface as reflected by the thickness histograms (Figure 4e,h,k). This phenomenon can be explained by the different variables that affect the formation and growth of polymeric brushes such as the type of catalyst, solvent, or ligand, the number of generated free radicals, or the use of a deactivator. ${ }^{48}$ For example, a heterogeneous distribution of the initiator over the fiber surface might lead to variable initiation and growth rates of the polymeric brush. Also, the living character of the grafted polymers might play a role in the development of the polymeric chains as evidenced by other groups as well. ${ }^{49}$

Furthermore, we studied the thickness of the brushes and their thermoresponsive behavior under wet conditions. Figure S2a shows an optical micrograph of a fiber immersed in $\mathrm{H}_{2} \mathrm{O}$ with an expanded PNIPAM brush. Supporting Video SV1 shows the expansion and compaction of the brush upon a temperature increase from 20 to $40{ }^{\circ} \mathrm{C}$. At $\sim 30{ }^{\circ} \mathrm{C}$, the hydrophilic-to-hydrophobic transition of the PNIPAM graft side chains was clearly observed, which demonstrates the 
thermoresponsive character of these PNIPAM-modified PVA microfibers qualitatively. Supporting Figure S2b presents quantitative data on the wet thickness of the brush on PVAPNIPAM 100 fibers as a function of the water temperature. The wet thickness of PNIPAM brushes decreased at temperatures above $\sim 30{ }^{\circ} \mathrm{C}$, followed by a slow recovery of the original brush thickness when the temperature was reduced again to $20{ }^{\circ} \mathrm{C}$. This thermoresponsive transition from hydrophilic to hydrophobic behavior of PNIPAM brushes grafted on solid PVA fibers is consistent with the LCST behavior of PNIPAM in solution. In solution, PNIPAM undergoes a coil-to-globule transition since intermolecular hydrogen bonding between PNIPAM chains and water molecules is replaced by intramolecular hydrogen bonding within PNIPAM chains when the temperature increases above the LCST. ${ }^{50,51}$ Polymer brushes grown onto solid surfaces often exhibit a broader LCST range than PNIPAM polymers in solution, which is in agreement with our LCST range of $\sim 30-$ $34{ }^{\circ} \mathrm{C}$ for PNIPAM brushes in comparison with $\sim 32-34{ }^{\circ} \mathrm{C}$ for bulk PNIPAM in solution. ${ }^{51}$

In $\mathrm{NaH}_{2} \mathrm{PO}_{4}$ solution, lower LCST temperatures of $\sim 24{ }^{\circ} \mathrm{C}$ were observed due to the presence of $\mathrm{H}_{2} \mathrm{PO}_{4}{ }^{-}$ions. Generally, ions are known to reduce the LCST as predicted by the Hofmeister series. The addition of salt ions results in a saltingout effect of the amide group, followed by an even stronger dehydration of the hydrophobic areas of the polymer. ${ }^{52}$ In our study, a 4 wt $\% \mathrm{NaH}_{2} \mathrm{PO}_{4}$ solution (corresponding to a concentration of $0.6 \mathrm{M}$ ) leads to an LCST value that is in agreement with the LCST of bulk PNIPAM in solution. ${ }^{52}$ Also, the thickness of the brush is lowered by almost $10 \%$ in saline solution versus distilled water. Since kosmotrope ion-water interactions are stronger than water-water interactions, the compaction of PNIPAM brushes upon temperature increase was more pronounced in $\mathrm{NaH}_{2} \mathrm{PO}_{4}$ than in distilled water. ${ }^{53}$

Supporting Figure S2c,d present histograms of the wet brush thickness of PVA-PNIPAM 50 and PVA-PNIPAM 100 fibers. Both PNIPAM-modified fibers swelled approximately threefold compared with their dry thickness in $\mathrm{H}_{2} \mathrm{O}$ at $24^{\circ} \mathrm{C}$.

The thermograms of PVA-PNIPAM 50 (Figure 5a) and PVA-PNIPAM 100 (Figure 5b) fibers are displayed as measured using DSC analysis. We observed that the thickness of the brushes started to decrease upon temperature increase at $\sim 24, \sim 28$, and $\sim 30{ }^{\circ} \mathrm{C}$ in $\mathrm{NaH}_{2} \mathrm{PO}_{4}, \mathrm{PBS}$, and $\mathrm{H}_{2} \mathrm{O}$, respectively, which is in line with the results displayed in Figure S2b.

The polarity of PNIPAM brushes on the surface of PVA fibers was characterized by meniscus contact angle measurements at 21 and $37{ }^{\circ} \mathrm{C}$ (Figure 5c). The unmodified PVA fibers showed the highest contact angle caused by the hydrophobic coating as present on the as-received PVA fibers. By treating PVA fibers with oxygen plasma or the THF solvent, we observed a significant decrease in the contact angle values caused by the removal of the hydrophobic surface coating from as-received PVA fibers.

Additionally, Figure S3b demonstrates that the PVA fiber presented a rather smooth surface with remnants of the hydrophobic coating still visible after the THF treatment. However, after the initiator attachment reaction, these remnants were not detected anymore. For the highest PNIPAM grafting density (PVA-PNIPAM 100), the contact angle values above $\left(37^{\circ} \mathrm{C}\right)$ and below $\left(21^{\circ} \mathrm{C}\right)$ the LCST were higher than PVA-PNIPAM 50 fibers and equal to values for the as-received PVA fiber (at $37^{\circ} \mathrm{C}$ ). Differences between the
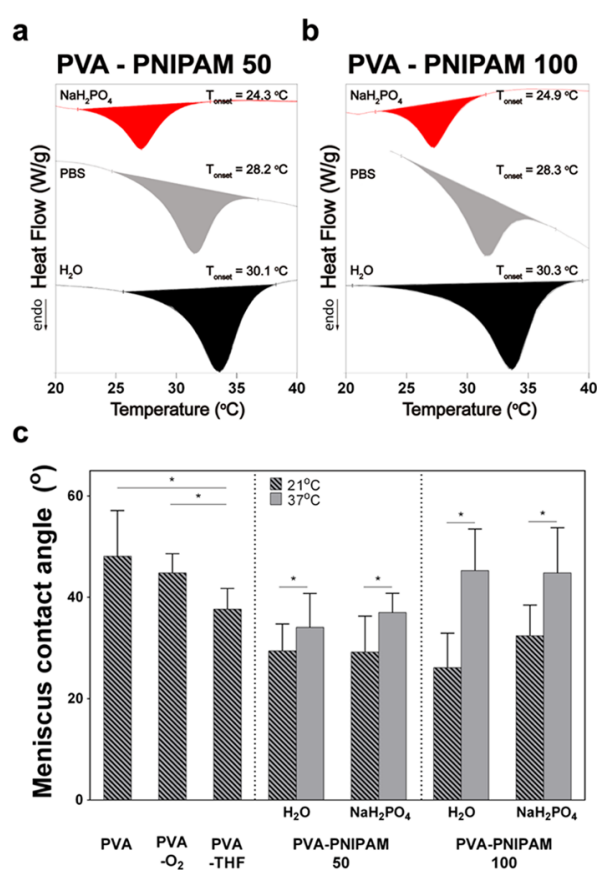

Figure 5. Characterization of PNIPAM-modified PVA fibers using DSC and contact angle measurements: (a, b) DSC analysis of PNIPAM-modified PVA fibers in $\mathrm{H}_{2} \mathrm{O}, \mathrm{PBS}$, and $\mathrm{NaH}_{2} \mathrm{PO}_{4}$; (c) meniscus contact angle measurements performed on pristine and PNIPAM-modified PVA fibers at 21 and $37{ }^{\circ} \mathrm{C}$ in $\mathrm{H}_{2} \mathrm{O}$ and $\mathrm{NaH}_{2} \mathrm{PO}_{4}$. Statistically significant differences are indicated with $*(p<$ $0.05)$.

contact angles recorded at 21 and $37{ }^{\circ} \mathrm{C}$ were statistically different, proving that the PNIPAM-modified PVA fibers are indeed thermoresponsive in both $\mathrm{H}_{2} \mathrm{O}$ and $\mathrm{NaH}_{2} \mathrm{PO}_{4}$.

To confirm if the chemical surface modifications did not reduce the mechanical strength of the PVA fibers, we also measured the tensile strength of as-received, THF-treated fibers and PNIPAM-modified PVA fibers (Figure S4). Evidently, the tensile strength of the PVA fiber was not affected by the chemical modifications.

The polymer chains were removed from the PVA fiber surface by base hydrolysis, after which the brush was analyzed by means of GPC. Figure S5 shows that the majority of the PNIPAM polymers were removed from the fiber surface, although few polymeric remnants were still observed. The obtained GPC results show a multimodal molecular weight distribution for the PNIPAM brushes, starting from a molecular weight of $2.9 \mathrm{~kg} / \mathrm{mol}$ for the lowest NIPAM/ CuBr-PMDETA ratio up to a maximum molecular weight of $150 \mathrm{~kg} / \mathrm{mol}$ for the PVA-PNIPAM 100 sample. Table S2 presents the grafting densities associated with the low and high molecular weights per sample as obtained from the cleavage of the polymeric brush. In view of the distribution of brush thicknesses as presented in Figure $4 \mathrm{i}-\mathrm{k}$, the multimodal molecular weight distribution was expected. However, the molecular weights obtained for the PVA-PNIPAM 100 sample are relatively low, which might be caused by technical limitations inherent to the GPC technique itself. Several studies indicated that PNIPAM is susceptible to adsorption to the stationary phase during GPC analysis. ${ }^{54}$ Moreover, filtration problems have been reported showing that strong chain entanglements resulted in retention of chains in the filter before entering the GPC column. ${ }^{55}$ Therefore, the GPC 
technique might be less suitable for analysis of high-molecularweight PNIPAM brushes, which might be more abundant for the PVA-PNIPAM 100 sample than currently detected.

We observed a higher fraction of low- versus high-molecularweight polymers as well as a decrease in the grafting density with increasing molecular weight. These results are in agreement with previous studies using different solid surfaces such as nanofibers, gold-coated slides, and nylon membranes. ${ }^{41,56,57}$ We speculate that these phenomena were related to the exothermic character of the polymerization reaction, which might have raised the temperature locally at the surface above the LCST, leading to PNIPAM aggregation and a decrease in the grafting density. Therefore, at early stages of the surface-initiated polymerization, many chains stop growing, which in turn reduces the grafting density of the brushes and leads to a heterogeneous chain growth. ${ }^{57}$ For the as-received PVA fiber, the GPC results showed that the hydrophobic coating removed via THF extraction had a molecular weight of $\sim 3 \mathrm{~kg} / \mathrm{mol}$, which was attributed to long-chain polyethylene waxes by Scheffler et al. ${ }^{58}$

3.2. Cytocompatibility of PNIPAM-Modified Fibers. A limitation of ATRP is the toxicity associated with the copperbased catalyst. Generally, the catalyst concentration should be at a level below $1 \mathrm{ppm}$ for biomedical applications. ${ }^{28}$ Figure S6 confirms that the amount of copper present on the fibers after the ATRP reaction was in the safe parts per billion range. In addition, since PNIPAM-modified PVA fibers were not used previously to reinforce CPCs, their cytocompatibility was tested in vitro. Figure S7 shows that the proliferation rate of cells (as expressed by their DNA content) cultured in the presence of coated and uncoated PVA fibers was comparable for all experimental groups. Moreover, we observed that cell proliferation was comparable to the positive (fiber-free) control, proving that none of the surface modifications hindered the proliferation of cultured cells. Consequently, it can be concluded that the PNIPAM brushes synthesized on the surface of PVA fibers were not cytotoxic.

Additionally, our group recently also studied the in vivo biocompatibility of both resorbable and nonresorbable PVA fiber-reinforced calcium phosphate cements. ${ }^{26}$ After 12 weeks of implantation, the PVA fibers were still clearly present, confirming that these fibers are nondegradable. A combination of stretching, heat treatment, and acetalization renders these high-tenacity, heavily cross-linked fibers ${ }^{59,60}$ nondegradable materials. Nevertheless, these fibers did not induce inflammatory responses or other adverse reactions when the fibers were well dispersed in the cement matrix. Moreover, the inclusion of PVA fibers did not negatively affect the ingrowth of new bone into the PVA fiber-reinforced cements.

3.3. Mechanical Properties of CPCs Reinforced with PNIPAM-Functionalized Microfibers. X-ray diffractograms confirmed that following the 3 day incubation in PBS, cements were converted to the apatite phase (Figure S8), as characterized by the main reflection peaks at 26 and $32^{\circ} 2 \theta$. The XRD diffractograms did not show any dissimilarities between samples with or without PVA or PNIPAM-modified PVA fibers. Consequently, it was concluded that PVA fibers with or without PNIPAM brushes did not negatively affect the chemical phase transformation of the $\alpha$-TCP precursor phase toward the apatite phase. Based on these results and previous findings from our group, ${ }^{9,61}$ we assume that the incorporation of PVA fibers did not negatively affect properties such as the setting times, injectability, and cohesion of the CPCs.
Nevertheless, these properties should be investigated in more detail in future studies.

Generally, CPCs fail suddenly in a brittle manner without any preceding deformation, whereas fiber addition can lead to increased ductility and toughness of the composite material. Composites can undergo three modes of failure: (i) brittle fracture, (ii) tension-softening for quasi-brittle materials, and (iii) strain-hardening for ductile materials. ${ }^{62}$

Fiber bridging, crack deflection, and frictional sliding are three mechanisms of fiber reinforcement that can be utilized in understanding the complex interactions between the fibers and the CPC matrix. Fiber bridging ${ }^{13}$ takes place whenever a crack in the composite material occurs and the fibers play the role of arresting the crack from further propagation. On the other hand, crack deflection refers to the distance of crack propagation resulting in enhanced energy dissipation. Finally, frictional sliding operates mainly at the interface between the fiber and the matrix and plays an important role in the effective stress transfer between the two constituents so that more energy is dissipated and less fractures occur. ${ }^{63}$

The pristine and PNIPAM-modified PVA fibers were added to CPCs at a $2.5 \%$ weight percentage, and their mechanical properties were tested in PBS at 21 and $37{ }^{\circ} \mathrm{C}$. Figure 6 presents the mechanical properties (flexural strength, modulus, and work of fracture) of CPCs reinforced with PVA fibers functionalized with PNIPAM brushes. The flexural strength of all experimental groups changed within a narrow range of 3.55.5 MPa. Compared with unreinforced CPC, flexural strength significantly increased for cements containing fibers of which the hydrophobic coating was removed using THF (Figure 6a). This treatment resulted in a 1.6-fold higher flexural strength of CPC-PVA-THF with respect to fiber-free CPCs. Similarly, the incorporation of THF-treated PVA fibers to the CPC matrix also enhanced the flexural modulus relative to pure CPC and CPC-PVA samples, resulting in 2-fold higher stiffness values. Functionalization of PVA fibers with PNIPAM at the highest molar NIPAM/CuBr-PMDETA ratio of 100 also led to a significantly increased flexural modulus compared with pure CPC and CPC-PVA composites, reaching stiffness values comparable to those of the CPC-PVA-THF specimens. No significant differences regarding flexural strength and flexural modulus were observed between composites tested at 21 and $37{ }^{\circ} \mathrm{C}$. Nevertheless, the unreinforced CPCs revealed work of fracture (WOF) values lower than $100 \mathrm{~J} / \mathrm{m}^{2}$, whereas the incorporation of PVA fibers improved the toughness of all fiber-reinforced composites considerably, as shown by WOF values higher than $1500 \mathrm{~J} / \mathrm{m}^{2}$ (Figure $6 \mathrm{c}$ ).

The highest WOF values were obtained for CPCs reinforced with either as-received PVA or PVA-PNIPAM 100-modified fibers. Interestingly, cements reinforced with PVA-PNIPAM 100 -modified fibers revealed significantly lower WOF values at $21{ }^{\circ} \mathrm{C}$ than at $37^{\circ} \mathrm{C}$. These results prove that the toughening effect of PNIPAM-modified fibers was 2-fold higher in their hydrophobic state. As-received PVA fibers were already covered by a hydrophobic proprietary coating. This coating was previously improved to optimize fiber reinforcement of concrete in civil engineering, but not for cements in biomedical engineering. Here, we have replaced the thermo-nonresponsive and potentially cytotoxic surface modification of as-received PVA fibers ${ }^{26}$ with a more cytocompatible and thermosensitive surface modification of comparable reinforcing efficacy.

The corresponding stress-strain curves shown in Figure S9 confirm the effective toughening of CPCs using PVA and 
a

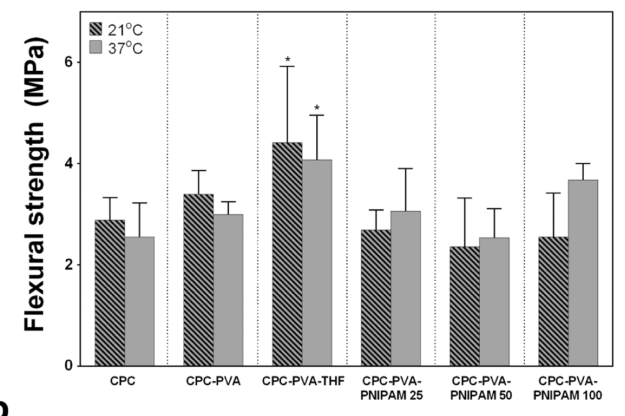

b
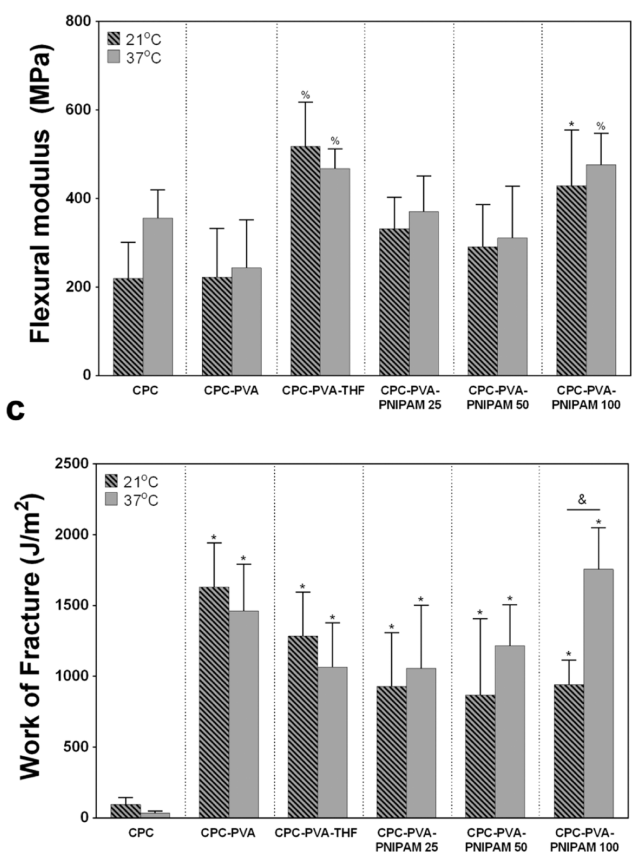

Figure 6. Mechanical properties of fiber-free and fiber-reinforced CPCs containing PNIPAM-modified PVA fibers: (a) flexural strength, (b) flexural modulus, and (c) work of fracture; statistically significant differences are indicated with * (vs CPC controls), \% (vs CPC-PVA), and $\&$ (among the same group) $(p<0.05)$.

PNIPAM-PVA fibers. Fiber-free CPCs revealed a clear brittle failure mode, whereas the CPC-PVA and CPC-PVA-THF curves follow a tension-softening behavior irrespective of the testing temperature. At $21{ }^{\circ} \mathrm{C}$, CPC-PVA-PNIPAM 100 samples reached the lowest yield stress, whereas the yield stress increased at $37{ }^{\circ} \mathrm{C}$, and stress-strain curves were indicative of strain-hardening behavior. For ceramic materials, this strain-hardening behavior obtained by fiber reinforcement is illustrative of ductile materials where the fibers are able to bridge and deflect propagating cracks.

Figure 7 shows typical single-fiber pullout force versus displacement curves for selected experimental groups. In Figure $7 \mathrm{a}$, it can be observed that the majority of as-received PVA fibers were pulled out from the matrix without rupturing after considerable frictional sliding, thereby facilitating energy dissipation required for efficient toughening of CPCs using PVA fibers. However, occasionally, a sudden partial drop in the pullout load was observed, which we attribute to a tunneling effect formed by the debonded fiber. Generally, the shape of the curves was comparable at both 21 and $37^{\circ} \mathrm{C}$. Figure $7 \mathrm{~b}$ presents the pullout curves of the THF solvent-treated PVA fibers where 13 out of 15 fibers ruptured after approximately
2-4 $\mathrm{mm}$ displacement. This effect was caused by the strong adhesion of these hydrophilic fibers to the cement matrix after removal of the hydrophobic surface coating. Moreover, PVATHF fibers displayed a higher aspect ratio due to the removal of the hydrophobic surface coating. The elastic modulus of cements is mainly determined by the initial part of the stressstrain curve before debonding of the fibers. On the contrary, toughness is mainly determined by frictional sliding at large strains after debonding of the fibers. Consequently, PVA-THF fibers contributed more to stiffening at low strains rather than toughening at large strains as compared with CPCs containing more hydrophobic PVA fibers.

Figure $7 \mathrm{c}, \mathrm{d}$ presents the pullout behavior of PVA-PNIPAM 100 fibers tested at either 21 or $37^{\circ} \mathrm{C}$ to assess the effect of the thermoresponsive polymeric PNIMPAM brushes covalently attached to the fiber surface. At $37^{\circ} \mathrm{C}$, most of the PNIPAMmodified fibers pulled out of the matrix, whereas the predominant pullout behavior at $21{ }^{\circ} \mathrm{C}$ was fiber rupture. These observations confirm that PNIPAM-modified fibers reinforced CPCs more efficiently in their collapsed but hydrophobic state, whereas the same fibers adhered too tightly to the CPC matrix at lower temperatures in their hydrophilic and expanded states.

Table 1 presents the average values of the maximum pullout force for all tested experimental groups. At $21{ }^{\circ} \mathrm{C}$, the maximum pullout load of PVA-PNIPAM 100 was $\sim 50 \%$ higher compared with the pullout force of hydrophobic as-received PVA fibers. The maximum pullout values were higher for PVAPNIPAM 100 versus PVA-PNIPAM 50 samples, which corresponds to the lower contact angle as measured for PVA-PNIPAM 100 fibers at $21^{\circ} \mathrm{C}$. At $37^{\circ} \mathrm{C}$, the maximum pullout force values of PVA-PNIPAM 100 fibers were also higher than those of the as-received PVA fibers. Since the contact angles of both types of PVA fibers were similar at 37 ${ }^{\circ} \mathrm{C}$, this phenomenon was most likely attributed to the higher fiber thickness and roughness of PNIPAM-modified fibers as compared with the smooth as-received PVA fibers.

To provide more insight into the mechanism of action of fiber reinforcement, Figure S10 presents the general pullout curves for PNIPAM-modified fibers at 21 and $37{ }^{\circ} \mathrm{C}$ in more detail. These pullout curves can be split into two parts, that is, a debonding and a pullout phase. As the load is applied, the fiber exhibits elastic behavior until maximum loading before initial debonding (indicated as point $\mathrm{P}_{\mathrm{a}}$ ). At this stage, the displacement corresponds to stretching of the fiber-free length and the partially debonded fiber part in the elastic region. ${ }^{64}$ Elastic deformation of PVA-PNIPAM fibers upon pullout prior to fiber debonding was similar at both testing temperatures, which explains why the stiffness of the PVA-PNIPAM fiberreinforced cements was comparable at 21 versus $37^{\circ} \mathrm{C}$. The sudden load drop from point $\mathrm{P}_{\mathrm{a}}$ to point $\mathrm{P}_{\mathrm{b}}$ reveals that debonding between the fiber and matrix took place. ${ }^{64}$ This can be considered as crack propagation at the fiber-cement matrix interface tunnel. ${ }^{65}$ After debonding, the fiber is gradually pulled out at larger displacements from the matrix by frictional sliding, as evidenced by several local increases in the pullout curve, which correspond to mechanical interlocking caused by surface deformations of the PNIPAM brushes. This phenomenon is observed for hooked or deformed fibers used in civil engineering. ${ }^{66}$ Evidently, PNIPAM-modified fibers pulled out at $21{ }^{\circ} \mathrm{C}$ ruptured when stresses exceeded the tensile strength of the fibers without contributing to frictional sliding, which contrasts with the pullout behavior of the same fibers at $37^{\circ} \mathrm{C}$ 

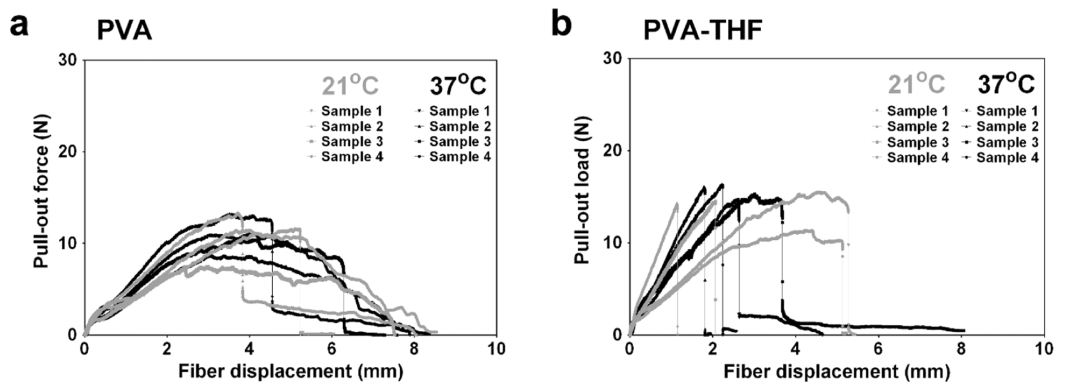

\section{C}

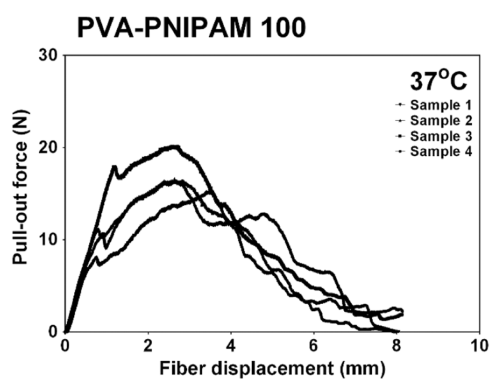

d

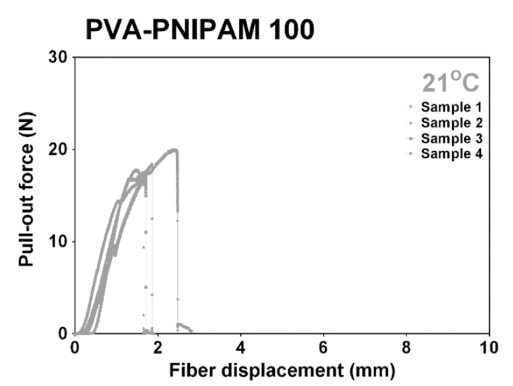

Figure 7. Force vs displacement curves for single-fiber pullout of PVA fibers from the CPC matrix: (a) PVA fibers pulled out at 21 and $37^{\circ} \mathrm{C}$, (b) PVA-THF fibers pulled out at 21 and $37^{\circ} \mathrm{C}$, (c) PVA-PNIPAM 100 fibers pulled out at $37^{\circ} \mathrm{C}$ or (d) at $21^{\circ} \mathrm{C}$.

Table 1. Average Pullout Values of Maximum Pullout Force and Pullout Work for the Groups Tested at 21 and $37{ }^{\circ} \mathrm{C}^{a}$

$\begin{array}{cccccc}\text { fiber } & & \text { PVA } & \text { PVA-THF } & \text { PVA-PNIPAM 50 } & \text { PVA-PNIPAM 100 } \\ \text { maximum pullout load (N) } & 21{ }^{\circ} \mathrm{C} & 11.8 \pm 2.2 & 15.0 \pm 1.7^{*} & 14.0 \pm 3.7 & 18.6 \pm 3.1^{*} \\ & 37^{\circ} \mathrm{C} & 11.9 \pm 1.9 & 14.7 \pm 2.6^{*} & 12.9 \pm 2.6 & 15.2 \pm 3.4^{*}\end{array}$

${ }^{a}$ Statistically significant differences compared with PVA fibers are indicated with an asterisk.

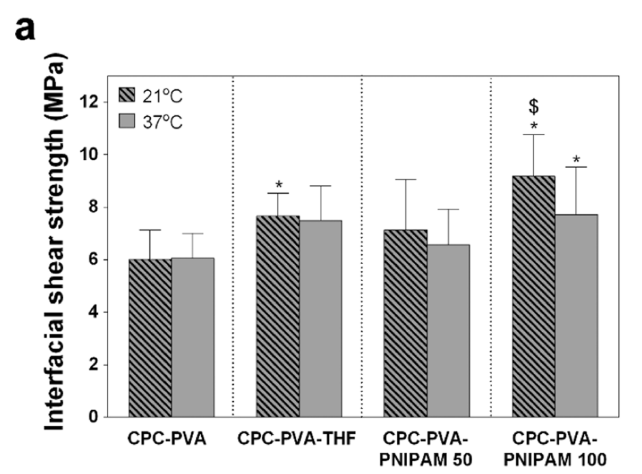

b

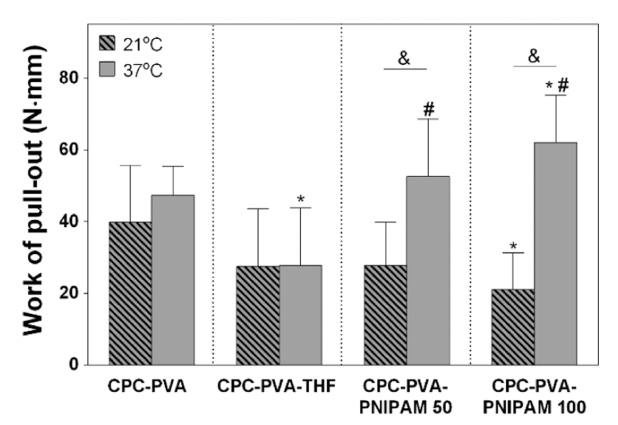

Figure 8. (a) Interfacial shear strength between CPC and pristine or surface-modified PVA fibers. (b) Work of pullout values resulting from the pullout test performed at 21 and $37^{\circ} \mathrm{C}$; statistically significant differences are indicated with * (vs CPC-PVA), \$ (vs CPC-PVA-PNIPAM 50), \# (vs CPC-PVA-THF), and \& (among the same group) $(p<0.05)$.

characterized by considerable frictional sliding. This observation can be explained by the hydrophobicity, as well as lower thickness and roughness of these fibers at $37^{\circ} \mathrm{C}$.

Furthermore, we calculated the average interfacial shear strength (IFSS) of the fibers and the cement matrix. The results in Figure 8a show that the average interfacial shear strength (IFSS) did not depend on temperature for CPCs containing pristine as-received PVA fibers. However, for PNIPAM-modified PVA fibers, we observed an increase in the IFSS values at a lower temperature of $21{ }^{\circ} \mathrm{C}$. At this temperature, the IFSS between CPC and PVA-PNIPAM 100 fibers was $40 \%$ higher compared with that of as-received PVA fibers. These results are in line with the contact angle measurements, which indicate that PVA-NIPAM 100 fibers were more hydrophilic than as-received PVA fibers, resulting in stronger adhesion to the CPC matrix. Consequently, hydrophobic as-received PVA fibers exhibited pullout behavior at 21 ${ }^{\circ} \mathrm{C}$, whereas the other three types of fibers were loaded until the tensile strength was exceeded, resulting in fiber rupture. At $37{ }^{\circ} \mathrm{C}$, the IFSS between CPC and PVA-PNIPAM 100 fibers was $\sim 7.5 \mathrm{MPa}$, whereas lower IFSS values were observed for as-received PVA fibers $(\sim 6 \mathrm{MPa})$, proving that the smooth hydrophobic coating of the as-received PVA fibers offered less resistance to pullout than the PNIPAM brushes as present on the PVA-PNIPAM 100 fiber.

Figure $8 \mathrm{~b}$ shows that the work of pullout (WOP) of PNIPAM-modified fibers was significantly lower at 21 versus $37^{\circ} \mathrm{C}$. WOP was 2 -fold higher at 37 versus $21{ }^{\circ} \mathrm{C}$ for PVA- 
PNIPAM 50 fibers and 3-fold higher for PVA-PNIPAM 100 fibers. PVA-PNIPAM 100 fibers revealed $30 \%$ higher WOP values than as-received PVA fibers, which means that the polymeric brushes effectively increased the amount of work needed for fiber pullout at $37^{\circ} \mathrm{C}$.

By comparing these results with those in Figure 6, we conclude that higher WOP values obtained using single-fiber pullout tests do not necessarily correspond to higher WOF values of fiber-reinforced CPCs since the WOF values of CPCPVA and CPC-PVA-PNIPAM 100 composites were similar. It should be stressed, however, that WOF is not an intrinsic material property but related to experimental parameters such as specimen geometry. ${ }^{62}$ Generally, large specimens such as the bars tested herein $\left(40 \times 40 \times 10 \mathrm{~cm}^{3}\right)$ are more sensitive to the formation of porosity due to the manual mixing of fibers, cement powder, and the liquid phase, which might have compromised the fiber reinforcement efficacy in these large samples.

Following the pullout tests, the fiber surface was investigated via SEM (Figure 9a,b). PVA and PVA-THF revealed clear

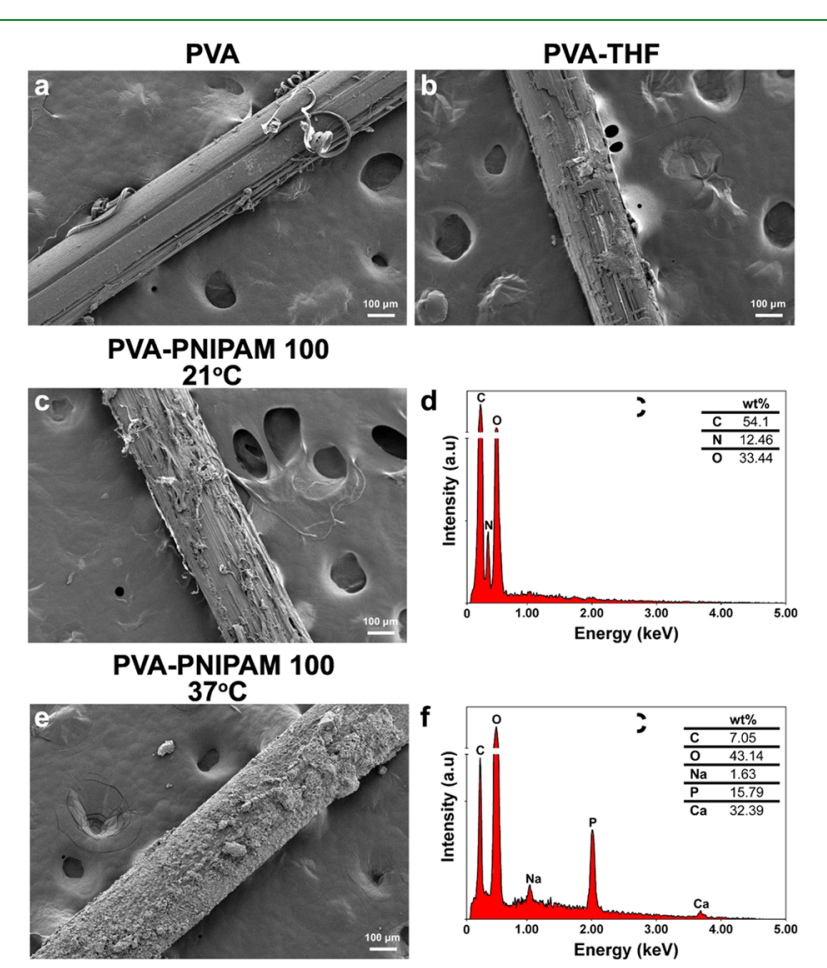

Figure 9. Scanning electron micrographs of PVA fibers after the pullout test: (a) as-received PVA fiber, (b) THF-treated PVA fiber, (c) PVA-PNIPAM 100 fiber after a pullout test performed at $21{ }^{\circ} \mathrm{C}$, and (d) the corresponding EDX spectrum, (e) PVA-PNIPAM 100 fiber after a pullout test performed at $37^{\circ} \mathrm{C}$, and (f) the corresponding EDX spectrum.

signs of fiber abrasion after pullout, which are indicative of interfacial slip and facilitated efficient dissipation and toughening of the CPC matrix. Figure $9 \mathrm{c}$ shows that polymeric brushes were torn apart and (partially) peeled off from PVAPNIPAM 100 fibers, which was supported by the EDX spectrum (Figure 9d), confirming that $\mathrm{C}, \mathrm{N}$, and $\mathrm{O}$ (corresponding to the PVA fiber and PNIPAM brushes) were present on the fiber surface after pullout without any coverage by calcium phosphate particles. The PNIPAM brushes were expanded in their hydrophilic state at $21{ }^{\circ} \mathrm{C}$, resulting in a low stiffness at this temperature. ${ }^{67,68}$ Consequently, strong entanglements between PNIPAM brushes and the CPC surface were formed, leading to retention of the brushes within the CPC matrix and delamination of the brushes from the underlying PVA substrate at $21{ }^{\circ} \mathrm{C}$.

At $37{ }^{\circ} \mathrm{C}$ (Figure 9e), on the other hand, the fibers were fully covered after pullout by a thick layer of calcium-deficient hydroxyapatite particles with a $\mathrm{Ca} / \mathrm{P}$ ratio of $\sim 1.6$ (Figure 9f). At this temperature, the PNIPAM brushes were collapsed in their hydrophobic state, resulting in increased frictional sliding and accumulation of hydroxyapatite particles on the surface of PNIPAM-modified PVA fibers upon fiber pullout.

Compared with the pristine PVA fiber where the smooth fiber morphology and hydrophobicity dictate energy dissipation through a frictional sliding mechanism, PVA-PNIPAM fibers allow for more energy to be dissipated across its interface as reflected in the IFSS values.

Nano-CT analysis was carried out after flexural testing of specimens to assess the dispersion of PVA (Supporting Video SV2) and PVA-PNIPAM 100 fibers (Supporting Video SV3) within the cement matrix in $3 \mathrm{D}$. The thermoresponsive fibers were homogenously distributed within the cement, as opposed to a more heterogeneous dispersion of hydrophobic asreceived PVA fibers, which showed more abundant fiber agglomeration. This observation proves that PNIPAMmodified fibers can be dispersed more effectively in their hydrophilic state at temperatures below their LCST.

Ultimately, we would like to finalize our discussion by providing a comparison of the mechanical data presented here to other relevant studies pertaining to fiber-reinforced CPCs. Since the lowest toughness values reported for the cortical bone are in the range of $1.5-15 \mathrm{~kJ} / \mathrm{m}^{2},{ }^{6969}$ the toughness values we obtained $\left(\sim 1.5 \mathrm{~kJ} / \mathrm{m}^{2}\right)$ are still on the lower range. However, it should be stressed that the relatively large dimensions of the fibers used in our study were selected to allow for experimental single-fiber pullout testing. Previously, we showed that toughness values as high as $8.7 \pm 2.5 \mathrm{~kJ} / \mathrm{m}^{2}$ were obtained by incorporating PVA fibers of a higher aspect ratio (diameter $26 \mu \mathrm{m}$, length $6 \mathrm{~mm}$ ) at $5 \mathrm{wt} \%$. These values are in the same range as the highest values reported in a review by Krüger and Groll, where values ranging between $\sim 0.01$ and $11 \mathrm{~kJ} / \mathrm{m}^{2}$ were reported. ${ }^{16}$ Generally, the highest values of $\sim 11$ $\mathrm{kJ} / \mathrm{m}^{2}$ were obtained for much higher fiber amounts of up to 45 vol \%. Summarizing, these results stress the remarkable efficacy of PVA fibers for reinforcement of CPCs. This considerable enhancement of the toughness and ductility of CPCs reinforced with PVA fibers can broaden their clinical applicability to more load-bearing defect sites where unreinforced CPCs do not provide the proper mechanical stability.

\section{CONCLUSIONS}

The present study investigated if modification of poly(vinyl alcohol) (PVA) fibers with thermoresponsive poly $(N$-isopropylacrylamide) (PNIPAM) brushes improves the efficacy of fiber reinforcement of calcium phosphate cements (CPCs). Thermoresponsive PNIPAM brushes of tunable thickness and lower critical solution temperature (LCST) were successfully attached onto PVA fibers through atom transfer radical polymerization. Dispersion of PNIPAM-modified PVA fibers was facilitated by their hydrophilicity below the LCST, whereas the work of single-fiber pullout and work of fracture increased considerably in their hydrophobic state above the 
LCST. These results confirm that the modification of polymeric fibers with thermoresponsive brushes represents an effective strategy to tune the dispersion and reinforcement efficacy of these fibers for the design of fiber-reinforced calcium phosphate cements with load-bearing properties.

Our approach is versatile and can be applied to other materials that are often used to reinforce CPCs such as glass, carbon, polypropylene, aramid, and polyamide fibers. This approach allows systematic investigation of the correlation between the fiber-matrix interface properties and the mechanical performance of fiber-reinforced $\mathrm{CaP}$ ceramics.

\section{ASSOCIATED CONTENT}

\section{S Supporting Information}

The Supporting Information is available free of charge on the ACS Publications website at DOI: 10.1021/acsami.9b08311.

Further experimental data including histograms on fiber length and brush thickness, SEM, XRD, GPC, stressstrain curves of fiber-reinforced composites, DNA quantification (PDF)

Videos showing 3D nano-CT videos of different fiberreinforced specimens (ZIP)

\section{AUTHOR INFORMATION}

\section{Corresponding Authors}

*E-mail: daniela.wilson@science.ru.nl (D.A.W.).

*E-mail: sander.leeuwenburgh@radboudumc.nl (S.C.G.L.).

\section{ORCID}

Daniela A. Wilson: 0000-0002-8796-2274

Sander C. G. Leeuwenburgh: 0000-0003-1471-6133

Notes

The authors declare no competing financial interest.

\section{ACKNOWLEDGMENTS}

The authors would like to thank Erik de Ronde for the practical assistance in DSC measurements and the collaborators at KU Leuven for the nano-CT images, which have been generated on the X-ray computed tomography facility of the Department of Development and Regeneration of the KU Leuven, financed by the Hercules foundation. D.-G.P., A.P., and S.C.G.L. acknowledge NWO Applied \& Engineering Sciences VIDI grant \#13455 from the Netherlands Organisation for Scientific Research (NWO). D.A.W. and Y.T. acknowledge NWO Chemische Wetenschappen VIDI Grant 723.015.001 and ERC Starting Grant 307679 StomaMotors for funding. R.N. acknowledges RIMLS grant \#014-057.

\section{REFERENCES}

(1) Fu, Q.; Saiz, E.; Rahaman, M. N.; Tomsia, A. P. Toward Strong and Tough Glass and Ceramic Scaffolds for Bone Repair. Adv. Funct. Mater. 2013, 23, 5461-5476.

(2) Larsson, S.; Hannink, G. Injectable Bone-Graft Substitutes: Current Products, Their Characteristics and Indications, and New Developments. Injury 2011, 42, S30-S34.

(3) Von Gonten, A. S.; Kelly, J. R.; Antonucci, J. M. Load-Bearing Behavior of a Simulated Craniofacial Structure Fabricated from a Hydroxyapatite Cement and Bioresorbable Fiber-Mesh. J. Mater. Sci.: Mater. Med. 2000, 11, 95-100.

(4) Barralet, J. E.; Tremayne, M.; Lilley, K. J.; Gbureck, U. Modification of Calcium Phosphate Cement with Alpha-Hydroxy Acids and Their Salts. Chem. Mater 2005, 17, 1313-1319.
(5) Engstrand, J.; Persson, C.; Engqvist, H. The Effect of Composition on Mechanical Properties of Brushite Cements. J. Mech. Behav. Biomed. Mater. 2014, 29, 81-90.

(6) Rödel, M.; Teßmar, J.; Groll, J.; Gbureck, U. Highly Flexible and Degradable Dual Setting Systems Based on Peg-Hydrogels and Brushite Cement. Acta Biomater. 2018, 79, 182-201.

(7) Abtahi, S. M.; Sheikhzadeh, M.; Hejazi, S. M. Fiber-Reinforced Asphalt-Concrete - a Review. Constr. Build. Mater. 2010, 24, 871877.

(8) Petre, D. G.; Kucko, N. W.; Abbadessa, A.; Vermonden, T.; Polini, A.; Leeuwenburgh, S. C. G. Surface Functionalization of Polylactic Acid Fibers with Alendronate Groups Does Not Improve the Mechanical Properties of Fiber-Reinforced Calcium Phosphate Cements. J. Mech. Behav. Biomed. Mater. 2019, 90, 472-483.

(9) Zuo, Y.; Yang, F.; Wolke, J. G. C.; Li, Y. B.; Jansen, J. A. Incorporation of Biodegradable Electrospun Fibers into Calcium Phosphate Cement for Bone Regeneration. Acta Biomater. 2010, 6, 1238-1247.

(10) Chew, K. K.; Low, K. L.; Sharif Zein, S. H.; McPhail, D. S.; Gerhardt, L. C.; Roether, J. A.; Boccaccini, A. R. Reinforcement of Calcium Phosphate Cement with Multi-Walled Carbon Nanotubes and Bovine Serum Albumin for Injectable Bone Substitute Applications. J. Mech. Behav. Biomed. Mater. 2011, 4, 331-339.

(11) Xu, H. H.; Eichmiller, F. C.; Giuseppetti, A. A. Reinforcement of a Self-Setting Calcium Phosphate Cement with Different Fibers. J. Biomed. Mater. Res. 2000, 52, 107-114.

(12) Maenz, S.; Kunisch, E.; Muhlstadt, M.; Bohm, A.; Kopsch, V.; Bossert, J.; Kinne, R. W.; Jandt, K. D. Enhanced Mechanical Properties of a Novel, Injectable, Fiber-Reinforced Brushite Cement. J. Mech. Behav. Biomed. Mater. 2014, 39, 328-338.

(13) $\mathrm{Xu}, \mathrm{H}$. H.; Eichmiller, F. C.; Barndt, P. R. Effects of Fiber Length and Volume Fraction on the Reinforcement of Calcium Phosphate Cement. J. Mater. Sci.: Mater. Med. 2001, 12, 57-65.

(14) Bentur, A.; Mindess, S. Fibre-Cement Interactions. Fibre Reinforced Cementitious Composites, 2nd ed.; Taylor \& Francis: Oxon, U.K., 2007; pp 31-104.

(15) Li, V. C. From Micromechanics to Structural Engineering: The Design of Cementitious Composite for Civil Engineering Applications. J. Struct. Mech. Earhquake Eng. 1993, 10, 37s-48s.

(16) Krüger, R.; Groll, J. Fiber Reinforced Calcium Phosphate Cements - on the Way to Degradable Load Bearing Bone Substitutes? Biomaterials 2012, 33, 5887-5900.

(17) Canal, C.; Gallinetti, S.; Ginebra, M. P. Low-Pressure Plasma Treatment of Polylactide Fibers for Enhanced Mechanical Performance of Fiber-Reinforced Calcium Phosphate Cements. Plasma Processes Polym. 2014, 11, 694-703.

(18) Maenz, S.; Hennig, M.; Muhlstadt, M.; Kunisch, E.; Bungartz, M.; Brinkmann, O.; Bossert, J.; Kinne, R. W.; Jandt, K. D. Effects of Oxygen Plasma Treatment on Interfacial Shear Strength and PostPeak Residual Strength of a PLGA Fiber-Reinforced Brushite Cement. J. Mech. Behav. Biomed. Mater. 2016, 57, 347-358.

(19) Gunnella, F.; Kunisch, E.; Bungartz, M.; Maenz, S.; Horbert, V.; Xin, L.; Mika, J.; Borowski, J.; Bischoff, S.; Schubert, H.; Hortschansky, P.; Sachse, A.; Illerhaus, B.; Gunster, J.; Bossert, J.; Jandt, K. D.; Ploger, F.; Kinne, R. W.; Brinkmann, O. Low-Dose Bmp2 Is Sufficient to Enhance the Bone Formation Induced by an Injectable, PLGA Fiber-Reinforced, Brushite-Forming Cement in a Sheep Defect Model of Lumbar Osteopenia. Spine J. 2017, 17, 16991711.

(20) Xu, H. H. K.; Quinn, J. B. Calcium Phosphate Cement Containing Resorbable Fibers for Short-Term Reinforcement and Macroporosity. Biomaterials 2002, 23, 193-202.

(21) Xu, B.; Toutanji, H. A.; Lavin, T.; Gilbert, J. A. Characterization of Poly(Vinyl Alcohol) Fiber Reinforced Organic Aggregate Cementitious Materials. Key Eng. Mater. 2011, 466, 73-83.

(22) Li, V. C.; Wang, S. X.; Wu, C. Tensile Strain-Hardening Behavior of Polyvinyl Alcohol Engineered Cementitious Composite (PVA-ECC). ACI Mater. J. 2001, 98, 483-492. 
(23) Li, V. C.; Wu, C.; Wang, S. X.; Ogawa, A.; Saito, T. Interface Tailoring for Strain-Hardening Polyvinyl Alcohol Engineered Cementitious Composite (PVA-ECC). ACI Mater. J. 2002, 99, 463-472.

(24) Redon, C.; Li, V. C.; Wu, C.; Hoshiro, H.; Saito, T.; Ogawa, A. Measuring and Modifying Interface Properties of PVA Fibers in ECC Matrix. J. Mater. Civil Eng. 2001, 12, 399.

(25) Ambrosio, L.; Guarino, V.; Sanginario, V.; Torricelli, P.; Fini, M.; Ginebra, M. P.; Planell, J. A.; Giardino, R. Injectable CalciumPhosphate-Based Composites for Skeletal Bone Treatments. Biomed. Mater. 2012, 7, No. 024113.

(26) Kucko, N. W.; Delacerda Schickert, S.; Sobral Marques, T.; Herber, R. P.; van den Beuken, J. J. J. P.; Zuo, Y.; Leeuwenburgh, S. C. G. Tough and Osteocompatible Calcium Phosphate Cements Reinforced with Poly(Vinyl Alcohol) Fibers. ACS Biomater. Sci. Eng. 2019, 5, 2491-2505.

(27) Perez, R. A.; Kim, H. W.; Ginebra, M. P. Polymeric Additives to Enhance the Functional Properties of Calcium Phosphate Cements. J. Tissue Eng. 2012, 3, No. 2041731412439555.

(28) Matyjaszewski, K. Atom Transfer Radical Polymerization (ATRP): Current Status and Future Perspectives. Macromolecules 2012, 45, 4015-4039.

(29) Spasojević, M.; Vorenkamp, J.; Jansen, M. R. P. A. C. S.; de Vos, P.; Schouten, A. J. Synthesis and Phase Behavior of Poly $(\mathrm{N}$ Isopropylacrylamide)-B-Poly(L-Lysine Hydrochloride) and Poly(NIsopropylacrylamide-Co-Acrylamide)-B-Poly(L-Lysine Hydrochloride). Materials 2014, 7, 5305-5326.

(30) Zoppe, J. O.; Habibi, Y.; Rojas, O. J.; Venditti, R. A.; Johansson, L. S.; Efimenko, K.; Osterberg, M.; Laine, J. Poly(N-Isopropylacrylamide) Brushes Grafted from Cellulose Nanocrystals Via SurfaceInitiated Single-Electron Transfer Living Radical Polymerization. Biomacromolecules 2010, 11, 2683-2691.

(31) Zoppe, J. O.; Osterberg, M.; Venditti, R. A.; Laine, J.; Rojas, O. J. Surface Interaction Forces of Cellulose Nanocrystals Grafted with Thermoresponsive Polymer Brushes. Biomacromolecules 2011, 12, $2788-2796$.

(32) Lin, H. C.; Hsieh, B. Z.; Lin, Y. L.; Sheng, Y. J.; Lin, J. J. Effect of Grafting Architecture on the Surfactant-Like Behavior of ClayPoly(NIPAAM) Nanohybrids. J. Colloid Interface Sci. 2012, 387, 106114.

(33) Gao, T.; Wang, X.; Yu, B.; Wei, Q.; Xia, Y.; Zhou, F. Noncovalent Microcontact Printing for Grafting Patterned Polymer Brushes on Graphene Films. Langmuir 2013, 29, 1054-1060.

(34) Ren, L. L.; Huang, S.; Zhang, C.; Wang, R. Y.; Tjiu, W. W.; Liu, T. X. Functionalization of Graphene and Grafting of TemperatureResponsive Surfaces from Graphene by ATRP "on Water". J. Nanopart. Res. 2012, 14, No. 940.

(35) Cao, L.; Man, T.; Zhuang, J. Q.; Kruk, M. Poly(NIsopropylacrylamide) and Poly(2-(Dimethylamino)Ethyl Methacrylate) Grafted on an Ordered Mesoporous Silica Surface Using Atom Transfer Radical Polymerization with Activators Regenerated by Electron Transfer. J. Mater. Chem. 2012, 22, 6939-6946.

(36) Shi, Y.; Selin, V.; Wang, Y.; Sukhishvili, S. A. Multiresponsive Block Copolymer-Modified "Hairy" Gold Nanoparticles for Remote Control of Interfaces. Part. Part. Syst. Charact. 2013, 30, 950-957.

(37) Gong, Z. L.; Tang, D. Y.; Guo, Y. D. The Fabrication and SelfFlocculation Effect of Hybrid $\mathrm{TiO}_{2}$ Nanoparticles Grafted with Poly(N-Isopropylacrylamide) at Ambient Temperature Via SurfaceInitiated Atom Transfer Radical Polymerization. J. Mater. Chem. 2012, $22,16872-16879$.

(38) Ionov, L. Soft Microorigami: Self-Folding Polymer Films. Soft Matter 2011, 7, 6786-6791.

(39) Cummings, C.; Murata, H.; Koepsel, R.; Russell, A. J. Tailoring Enzyme Activity and Stability Using Polymer-Based Protein Engineering. Biomaterials 2013, 34, 7437-7443.

(40) Tu, Y.; Peng, F.; Sui, X.; Men, Y.; White, P. B.; van Hest, J. C. M.; Wilson, D. A. Self-Propelled Supramolecular Nanomotors with Temperature-Responsive Speed Regulation. Nat. Chem. 2017, 9, 480.
(41) Gao, G.; Dallmeyer, J. I.; Kadla, J. F. Synthesis of Lignin Nanofibers with Ionic-Responsive Shells: Water-Expandable LigninBased Nanofibrous Mats. Biomacromolecules 2012, 13, 3602-3610.

(42) Gao, G. Z.; Karaaslan, M. A.; Kadla, J. F.; Ko, F. Enzymatic Synthesis of Ionic Responsive Lignin Nanofibres through Surface Poly(N-Isopropylacrylamide) Immobilization. Green Chem. 2014, 16, 3890-3898.

(43) Özçam, A. E.; Roskov, K. E.; Genzer, J.; Spontak, R. J. Responsive Pet Nano/Microfibers Via Surface-Initiated Polymerization. ACS Appl. Mater. Interfaces 2012, 4, 59-64.

(44) Yu, Y.; Kieviet, B. D.; Liu, F.; Siretanu, I.; Kutnyánszky, E.; Vancso, G. J.; de Beer, S. Stretching of Collapsed Polymers Causes an Enhanced Dissipative Response of Pnipam Brushes near Their Lcst. Soft Matter 2015, 11, 8508-8516.

(45) Chen, J. P.; Su, C. H. Surface Modification of Electrospun Plla Nanofibers by Plasma Treatment and Cationized Gelatin Immobilization for Cartilage Tissue Engineering. Acta Biomater. 2011, 7, 234243

(46) Sanchis, M. R.; Blanes, V.; Blanes, M.; Garcia, D.; Balart, R. Surface Modification of Low Density Polyethylene (LDPE) Film by Low Pressure O-2 Plasma Treatment. Eur. Polym. J. 2006, 42, 15581568.

(47) Kim, K. S.; Lee, K. H.; Cho, K.; Park, C. E. Surface Modification of Polysulfone Ultrafiltration Membrane by Oxygen Plasma Treatment. J. Membr. Sci. 2002, 199, 135-145.

(48) Greszta, D.; Mardare, D.; Matyjaszewski, K. "Living” Radical Polymerization. I. Possibilities and Limitations. Macromolecules 1994, 27, 638-644.

(49) Kong, H.; Gao, C.; Yan, D. Y. Functionalization of Multiwalled Carbon Nanotubes by Atom Transfer Radical Polymerization and Defunctionalization of the Products. Macromolecules 2004, 37, 40224030.

(50) Rahane, S. B.; Floyd, J. A.; Metters, A. T.; Kilbey, S. M. Swelling Behavior of Multiresponsive Poly(Methacrylic Acid)-BlockPoly(N-Isopropylacrylamide) Brushes Synthesized Using SurfaceInitiated Photoiniferter-Mediated Photopolymerization. Adv. Funct. Mater. 2008, 18, 1232-1240.

(51) Wang, S. Q.; Zhu, Y. X. Facile Method to Prepare Smooth and Homogeneous Polymer Brush Surfaces of Varied Brush Thickness and Grafting Density. Langmuir 2009, 25, 13448-13455.

(52) Zhang, Y.; Furyk, S.; Sagle, L. B.; Cho, Y.; Bergbreiter, D. E.; Cremer, P. S. Effects of Hofmeister Anions on the LCST of PNIPAM as a Function of Molecular Weight. J. Phys. Chem. C 2007, 111, $8916-8924$

(53) Moghaddam, S. Z.; Thormann, E. Hofmeister Effect on Pnipam in Bulk and at an Interface: Surface Partitioning of Weakly Hydrated Anions. Langmuir 2017, 33, 4806-4815.

(54) Swift, T.; Hoskins, R.; Telford, R.; Plenderleith, R.; Pownall, D.; Rimmer, S. Analysis Using Size Exclusion Chromatography of Poly(N-Isopropyl Acrylamide) Using Methanol as an Eluent. J. Chromatogr. A 2017, 1508, 16-23.

(55) Schild, H. G. Poly(N-Isopropylacrylamide) - Experiment, Theory and Application. Prog. Polym. Sci. 1992, 17, 163-249.

(56) Plunkett, K. N.; Zhu, X.; Moore, J. S.; Leckband, D. E. Pnipam Chain Collapse Depends on the Molecular Weight and Grafting Density. Langmuir 2006, 22, 4259-4266.

(57) Martinez, A. P.; Carrillo, J.-M. Y.; Dobrynin, A. V.; Adamson, D. H. Distribution of Chains in Polymer Brushes Produced by a "Grafting from" Mechanism. Macromolecules 2016, 49, 547-553.

(58) Scheffler, C.; Zhandarov, S.; Jenschke, W.; Mader, E. Poly(Vinyl Alcohol) Fiber Reinforced Concrete: Investigation of Strain Rate Dependent Interphase Behavior with Single Fiber Pullout Test under Quasi-Static and High Rate Loading. J. Adhes. Sci. Technol. 2013, 27, 385-402.

(59) Mark, H. F. Encyclopedia of Polymer Science and Technology, 3rd ed.; John Wiley and Sons: New York, 2005; Vol. 8.

(60) Hiroyoshi, T.; Mitsuo, S.; Fujio, U. Ultra-High-Tenacity Polyvinyl Alcohol Fiber and Process for Producing Same. EP0146084A3, 1986 
(61) Castro, A. G. B.; Polini, A.; Azami, Z.; Leeuwenburgh, S. C. G.; Jansen, J. A.; Yang, F.; van den Beucken, J. Incorporation of Plla Micro-Fillers for Mechanical Reinforcement of Calcium-Phosphate Cement. J. Mech. Behav. Biomed. Mater. 2017, 71, 286-294.

(62) Zhang, J. T.; Liu, W. Z.; Schnitzler, V.; Tancret, F.; Bouler, J. M. Calcium Phosphate Cements for Bone Substitution: Chemistry, Handling and Mechanical Properties. Acta Biomater. 2014, 10, 10351049.

(63) Polini, A.; Petre, D. G.; Iafisco, M.; de Lacerda Schickert, S.; Tampieri, A.; van den Beucken, J.; Leeuwenburgh, S. C. G. Polyester Fibers Can Be Rendered Calcium Phosphate-Binding by Surface Functionalization with Bisphosphonate Groups. J. Biomed. Mater. Res., Part A 2017, 105, 2335-2342.

(64) Arain, M. F.; Wang, M. X.; Chen, J. Y.; Zhang, H. P. Study on PVA Fiber Surface Modification for Strain-Hardening Cementitious Composites (PVA-SHCC). Constr. Build. Mater. 2019, 197, 107-116. (65) Lin, Z.; Li, V. C. Crack Bridging in Fiber Reinforced Cementitious Composites with Slip-Hardening Interfaces. J. Mech. Phys. Solids 1997, 45, 763-787.

(66) Hamoush, S.; Abu-Lebdeh, T.; Cummins, T.; Zornig, B. Pullout Characterizations of Various Steel Fibers Embedded in Very HighStrength Concrete. Am. J. Eng. Appl. Sci. 2010, 3, 418-426.

(67) Schmidt, S.; Zeiser, M.; Hellweg, T.; Duschl, C.; Fery, A.; Mohwald, H. Adhesion and Mechanical Properties of PNIPAM Microgel Films and Their Potential Use as Switchable Cell Culture Substrates. Adv. Funct. Mater. 2010, 20, 3235-3243.

(68) Sui, X. F.; Chen, Q.; Hempenius, M. A.; Vancso, G. J. Probing the Collapse Dynamics of Poly(N-Isopropylacrylamide) Brushes by AFM: Effects of Co-Nonsolvency and Grafting Densities. Small 2011, 7, 1440-1447.

(69) Currey, J. D.; Butler, G. The Mechanical Properties of Bone Tissue in Children. J. Bone Jt. Surg. 1975, 57, 810-814. 